DOI: $\mathrm{xxx} / \mathrm{xxxx}$

\title{
Numerical Design of Lyapunov Functions for a Class of
}

\section{Homogeneous Discontinuous Systems ${ }^{\dagger}$}

\author{
Jesús Mendoza-Avila*1 | Denis Efimov ${ }^{2,3}$ | Rosane Ushirobira ${ }^{2}$ | Jaime A. Moreno ${ }^{4}$
}

${ }^{1}$ Facultad de Ingeniería,Universidad Nacional Autónoma de México,Mexico City, Mexico.

${ }^{2}$ Inria, Univ. Lille, CNRS UMR 9189 CRIStAL, F-59000 Lille, France.

${ }^{3}$ Department of Control Systems and Informatics, University ITMO,Saint Petersburg, Russia

${ }^{4}$ Instituto de Ingeniería,Universidad Nacional Autónoma de México,Mexico City, Mexico.

\section{Correspondence}

Jesús Mendoza-Avila, Facultad de Ingeniería, Building T, Universidad Nacional Autónoma de México, 04510 Mexico City, Mexico, Email: jesus.menav.14@gmail.com

\begin{abstract}
Summary
This paper deals with the analytic and numeric design of a Lyapunov function for homogeneous and discontinuous systems. First, the presented converse theorems provide two analytic expressions of homogeneous and locally Lipschitz continuous Lyapunov functions for homogeneous discontinuous systems of negative homogeneity degree, generalizing classical results. Second, a methodology for the numerical construction of those Lyapunov functions is extended to the class of systems under consideration. Finally, the developed theory is applied to the numerical design of a Lyapunov function for some Higher-Order Sliding Mode algorithms.
\end{abstract}

\section{KEYWORDS:}

Lyapunov functions, Homogeneity, Differential inclusions, Nonlinear systems, Discontinuous control.

\section{1 | INTRODUCTION}

The Lyapunov function method is one of the main approaches to analyze the stability of dynamical systems ${ }^{1,2}$. For linear systems, the design of Lyapunov functions has already been widely studied ${ }^{1}$. In the case of nonlinear systems, a general methodology to design Lyapunov functions is still an open problem. However, several converse Lyapunov theorems have been

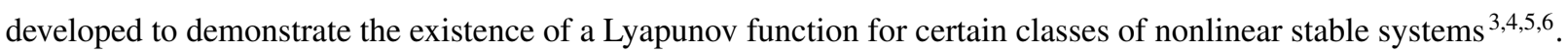

For instance, Clarke et al., ${ }^{7}$ present a converse Lyapunov theorem for multivalued systems. That paper shows the construction of a smooth Lyapunov function for Filippov differential inclusions generalizing the results of Kurzweil ${ }^{3}$, which is an extension of the results of Massera ${ }^{4}$ for the case of smooth vector fields. A remarkable fact in ${ }^{7}$ is that only the upper semi-continuous behavior of the Filippov inclusion is required for the existence of a smooth Lyapunov function for set-valued systems. Moreover,

\footnotetext{
${ }^{\dagger}$ This work was supported in part by Homogeneity Tools for Sliding Mode Control and Estimation (HoTSMoCE) INRIA associate team program; by CONACyT (Consejo Nacional de Ciencia y Tecnología) project 282013 and CVU 711867; by PAPIIT-UNAM (Programa de Apoyo a Proyectos de Investigación e Innovación Tecnológica) IN 115419 and IN 110719.
} 
Rosier ${ }^{8}$ presented other inverse Lyapunov theorem for discontinuous systems, where a smooth Lyapunov function is provided and Lyapunov and Lagrange stabilities are studied. The counterpart of the work in ${ }^{7}$ for discrete-time systems was developed by Kellet and Teel ${ }^{9}$, where the existence of smooth Lyapunov functions for robust globally asymptotically stable difference inclusions was proven.

Homogeneous systems ${ }^{1,2}$ constitute a subclass of nonlinear dynamics, possessing interesting properties, e.g., scalability of solutions, non-asymptotic rates of convergence, robustness. Such properties have been found advantageous for the design of homogeneous Lyapunov functions ${ }^{2,10}$. For homogeneous systems of positive degree, in Efimov et al. ${ }^{11}$ several explicit formulas of homogeneous Lyapunov functions are provided based on the converse theorems of Massera ${ }^{4}$ and Persidskii ${ }^{5}$. Moreover, Efimov et al. ${ }^{11}$ give a methodology for numerical construction of such functions. With a different approach, Sanchez and Moreno ${ }^{12,13}$ uses generalized homogeneous polynomials as Lyapunov functions for a class of homogeneous systems including negative homogeneity degree and discontinuous ones.

Homogeneous Lyapunov functions for discontinuous homogeneous systems have been studied by Nakamura et al. ${ }^{14}$. That paper unifies the converse theorems presented by Clarke et al. ${ }^{7}$ and Rosier ${ }^{8}$ into a simple and elegant framework with posterior construction of a homogeneous Lyapunov function associated with a homogeneous differential inclusion. In addition, the relationship between the homogeneity degree and the rate of convergence is also provided in Nakamura et al. ${ }^{14}$. Such a converse theorem was generalized by Tuna and Teel ${ }^{15}$ for homogeneous hybrid systems covering the discrete-time case as well.

Many examples of homogeneous discontinuous systems can be found in the family of Higher-Order Sliding Mode (HOSM) algorithms ${ }^{16}$. For a chain of integrators of order $n$, such algorithms are able to provide finite-time convergence to the $n$-th order sliding mode, i.e., to nullify the output $\sigma(t)$ and its $(n-1)$ derivatives $\left(\sigma(t)=\dot{\sigma}(t)=\ldots=\sigma^{(n-1)}(t)=0\right)$, even in presence of bounded non-vanishing matched disturbances or uncertainties ${ }^{16}$. Many approaches of Lyapunov function design for particular cases of HOSM algorithms have been developed.

For instance, generalized polynomial Lyapunov functions for homogeneous HOSM and continuous HOSM described by generalized forms ${ }^{1}$ are presented by Sanchez and Moreno ${ }^{12,13}$. A family of strict Lyapunov functions for the Super-Twisting Algorithm is provided by Moreno and Osorio ${ }^{17}$ by means of quadratic forms. Cruz-Zavala and Moreno ${ }^{18}$ proposed the design of a family HOSM controllers by means of homogeneous control Lyapunov functions. Polyakov and Poznyak ${ }^{19}$ introduced a Lyapunov function for the Twisting Algorithm based on the method of Zubov. A unified Lyapunov function for second order sliding mode control systems is presented by Polyakov and Poznyak ${ }^{20}$. The design of finite-time convergent controllers using the implicit Lyapunov function approach is introduced by Polyakov et al. ${ }^{21}$, where quasi-continuous HOSM appears as a particular case. For a class of HOSM described by homogeneous piecewise continuous vector fields, Sanchez and Moreno 22,23

\footnotetext{
${ }^{1}$ A generalized form is a homogeneous function $g: \mathbb{R}^{n} \rightarrow \mathbb{R}$ of degree $m \in \mathbb{R}$, with weights $r=\left[r_{1}, \ldots, r_{n}\right], r_{i}>0$, which consists of a linear combination of products of terms like $\left\lceil x_{i}\right\rfloor^{p}$ or $\left|x_{i}\right|^{q}$, where $p, q>0$ and $i \in\{1, \ldots, n\}$.
} 
introduced a constructive method to design homogeneous Lyapunov functions by integrating a positive definite function along the whole trajectories of the system. The latter method allows the design of a Lyapunov function for Twisting and Terminal algorithms but its extension to a broader class of systems is complicated.

Despite the number of papers in the literature related to converse Lyapunov theorems for homogeneous discontinuous systems, only the existence of the Lyapunov functions is proven in most of them under some restrictions. Contrarily, the present work provides explicit formulas of homogeneous Lipschitz continuous Lyapunov functions for discontinuous homogeneous systems basing on the converse theorems of Kurzweil, ${ }^{3}$ Massera $^{4}$, Persidskii ${ }^{5}$ and Yoshizawa ${ }^{6}$. Consequently, our results extend the methodology of numerical construction of homogeneous Lyapunov functions developed by Efimov et al. ${ }^{11}$ to a class of discontinuous homogeneous systems of negative degree, including several HOSM algorithms. The novelty of this paper is highlighted as follows:

- Two converse Lyapunov theorems are presented for homogeneous discontinuous systems, developing and generalizing previous results and also, providing explicit formulas of Lyapunov functions.

- The methodology of numerical design of Lyapunov functions is extended to cover the case of discontinuous homogeneous systems of negative degree.

- Both previous contributions establish a new framework to the numerical design of homogeneous and Lipschitz continuous Lyapunov functions for a wide class of HOSM.

The rest of the paper has the following structure. Section 2 introduces the notation used in the paper. In Section 3, some useful definitions and preparatory results are presented. Section 4 contains the converse theorems and Lyapunov function design for homogeneous discontinuous systems. Section 5 describes the methodology for a numerical construction of a Lyapunov function for homogeneous discontinuous systems. In Section 6, the proposed methodology is applied to the numerical design of a Lyapunov function for Twisting and Terminal algorithms. Finally, the conclusions are presented in Section 7.

\section{2 | NOTATION}

- $\mathbb{N}$ and $\mathbb{R}$ are the sets of natural and real numbers, respectively. Moreover, $\mathbb{R}_{+}$represents the set of nonnegative real numbers, i.e., $\mathbb{R}_{+}=\{x \in \mathbb{R}: x \geq 0\}$.

- $|\cdot|$ denotes the absolute value in $\mathbb{R},\|\cdot\|$ stands for the Euclidean norm in $\mathbb{R}^{n}$.

- For $x \in \mathbb{R}$ and $\gamma \in \mathbb{R}$, define $\lceil x\rfloor^{\gamma}:=|x|^{\gamma} \operatorname{sign}(\mathrm{x})$. 
- A continuous function $\alpha: \mathbb{R}_{+} \rightarrow \mathbb{R}_{+}$belongs to the class $\mathcal{K}$ if $\alpha(0)=0$ and it is strictly increasing. The function $\alpha: \mathbb{R}_{+} \rightarrow \mathbb{R}_{+}$belongs to the class $\mathcal{K}_{\infty}$ if $\alpha \in \mathcal{K}$ and it increases to infinity. A continuous function $\beta: \mathbb{R}_{+} \times \mathbb{R}_{+} \rightarrow \mathbb{R}_{+}$ belongs to the class $\mathcal{K} \mathcal{L}$ if $\beta(\cdot, t) \in \mathcal{K}_{\infty}$ for each fixed $t \in \mathbb{R}_{+}$and $\lim _{t \rightarrow \infty} \beta(s, t)=0$ for each fixed $s \in \mathbb{R}_{+}$.

- The boundary of a set $D$ is denoted by $\partial(D)$.

\section{3 | PRELIMINARIES}

Following the book of Filippov ${ }^{24}$ (see also the paper of Cortés ${ }^{25}$ ), a discontinuous vector field $f: \mathbb{R}^{n} \rightarrow \mathbb{R}^{n}$ is called piecewise continuous if there exist a finite number of disjoint, open and connected sets $D_{i} \subset \mathbb{R}^{n} ; i=1, \ldots, m$ whose closures $\bar{D}_{i}$ cover $\mathbb{R}^{n}$, i.e., $\mathbb{R}^{n}=\cup_{i=1}^{m} \bar{D}_{i}$, such that, for all $i=1, \ldots, m$, the vector field is continuous on $D_{i}$.

Consider the system

$$
\dot{x}(t)=f(x(t)), \quad t \in \mathbb{R}_{+},
$$

where $x(t) \in \mathbb{R}^{n}$ is the state and $f: \mathbb{R}^{n} \rightarrow \mathbb{R}^{n}$ is a piecewise continuous vector field and $f(0)=0$, i.e., the origin is an equilibrium point for the system (1). In the sequel, we will sometimes write $x \in \mathbb{R}^{n}$ for $x(t) \in \mathbb{R}^{n}$.

Let $S$ be the set of points where $f$ is discontinuous, such that $S$ is equal to the union of the boundaries of the sets $D_{i}$, i.e., $S=\cup_{i=1}^{m} \partial\left(D_{i}\right)$. Note that $S$ has measure zero.

For a piecewise continuous vector field $f: \mathbb{R}^{n} \rightarrow \mathbb{R}^{n}$, define the Filippov set-valued map $F: \mathbb{R}^{n} \rightrightarrows \mathbb{R}^{n}$ by

$$
F(x)=\overline{\operatorname{co}}\left\{\lim _{i \rightarrow \infty} f\left(x_{i}\right): x_{i} \rightarrow x, x_{i} \notin S\right\}, \quad \forall x \in \mathbb{R}^{n}
$$

where co denotes the convex closure of a set. The set $F(x)$ is non-empty, compact and convex for all $x \in \mathbb{R}^{n}$ and the set-valued function $F$ is upper semi-continuous ${ }^{24,25}$.

For any initial condition $x_{0} \in \mathbb{R}^{n}$ and $t \in\left[0, t_{1}\right] \subset \mathbb{R}_{+}$with $t_{1}>0$, a Filippov solution of the piecewise continuous system (1) is an absolutely continuous functions $\chi:\left[0, t_{1}\right] \times \mathbb{R}^{n} \rightarrow \mathbb{R}^{n}$ satisfying the inclusion $\dot{\chi}\left(t, x_{0}\right) \in F\left(\chi\left(t, x_{0}\right)\right)$ for almost all $t \in\left[0, t_{1}\right]$. A sufficient condition for uniqueness of solutions of a piecewise continuous system is given as follows

Proposition 1. (Filippov ${ }^{24}$ ). Let $f: \mathbb{R}^{n} \rightarrow \mathbb{R}^{n}$ be a piecewise continuous vector field, with $\mathbb{R}^{n}=\bar{D}_{1} \cup \bar{D}_{2}$ for disjoint, open and connected sets $D_{1}, D_{2} \subset \mathbb{R}^{n}$. Let $S=\partial\left(D_{1}\right)=\partial\left(D_{2}\right)$ be the set of points at which $f$ is discontinuous and assume that $S$ is a $C^{2}$-manifold. Moreover, assume that for $i=1,2$, the restriction of $f$ to $D_{i}$ admits a continuous extension to the closure $\bar{D}_{i}$ denoted by $f_{\mid \bar{D}_{i}}$, which is continuously differentiable on $D_{i}$ and $f_{\mid \bar{D}_{1}}-f_{\mid \bar{D}_{2}}$ is continuously differentiable on $S$. If for each $x \in S$, either $f_{\mid \bar{D}_{1}}(x)$ points into $D_{2}$ or $f_{\mid \bar{D}_{2}}(x)$ points into $D_{1}$, then $\chi\left(t, x_{0}\right)$ is the unique Filippov solution of (1) starting from any initial condition $x_{0} \in \mathbb{R}^{n}$. 
It is easy to check that the number of partitions $D_{i} \subset \mathbb{R}^{n} ; i=1, \ldots, m$ can be extended to any arbitrary finite number $m \geq 2$. Roughly speaking, the uniqueness of solutions on each set $D_{i}$ is warranted by the continuous differentiability of $f$ on $D_{i}$ while the assumption of continuous differentiability of $f_{\mid \bar{D}_{i}}-f_{\mid \bar{D}_{j}}$ along $S$, with $i \neq j$ and $i, j \in[1, m]$, guarantees that the uniqueness is not disrupted by the discontinuity 25 .

At the points belonging to $S$, the Filippov solution of the piecewise continuous system (1) can exhibit different behaviors. Suppose that $x \in S$ belongs to the boundary of just two sets, i.e., $x \in \partial\left(D_{i}\right) \cap \partial\left(D_{j}\right)$, for some $i, j \in[1, m]$, but $x \notin \partial\left(D_{k}\right)$, for all $k \in[1, m] \backslash\{i, j\}$. First, if all vectors belonging to $F(x)$ point into $D_{i}$ (respectively, $D_{j}$ ), then a Filippov solution that reaches $S$ at $x$ continues its motion in $D_{i}$ (respectively $D_{j}$ ). Second, if a vector in $F(x)$ is tangent to $S$, then either all Filippov solutions that start at $x$ leave $S$ immediately, or there exist Filippov solutions that reach the set $S$ at $x$ and slide on $S$ afterwards, which is called sliding motions ${ }^{25}$.

\section{1 | Weighted homogeneity}

Following the works in ${ }^{1,2,26,27}$, for strictly positive real numbers $r_{i}(i=1, \ldots, n)$ called weights and $\lambda>0$, define

- the vector of weights $r=\left(r_{1}, \ldots, r_{n}\right)^{T}, r_{\max }=\max _{1 \leq j \leq n} r_{j}$ and $r_{\min }=\min _{1 \leq j \leq n} r_{j}$;

- the dilation matrix $\Lambda_{r}(\lambda)=\operatorname{diag}\left(\lambda^{r_{i}}\right)_{i=1}^{n}$ such that, for all $x \in \mathbb{R}^{n}$ and for all $\lambda>0$,

$$
\Lambda_{r}(\lambda) x=\left(\lambda^{r_{1}} x_{1}, \ldots, \lambda^{r_{i}} x_{i}, \ldots, \lambda^{r_{n}} x_{n}\right)^{T}
$$

- the $r$-homogeneous norm of $x \in \mathbb{R}^{n}$ is given by $\|x\|_{r}=\left(\sum_{i=1}^{n}\left|x_{i}\right|^{\frac{\rho}{r_{i}}}\right)^{\frac{1}{\rho}}$ for $\rho \geq r_{\max }{ }^{2}$;

- for $s>0$, the sphere and the ball in the homogeneous norm are defined as $S_{r}(s)=\left\{x \in \mathbb{R}^{n}:\|x\|_{r}=s\right\}$ and $B_{r}(s)=$ $\left\{x \in \mathbb{R}^{n}:\|x\|_{r} \leq s\right\}$, respectively.

Definition 1. A function $g: \mathbb{R}^{n} \rightarrow \mathbb{R}$ is $r$-homogeneous of degree $\mu \in \mathbb{R}$ if for all $\lambda>0$ and all $x \in \mathbb{R}^{n}$,

$$
\lambda^{-\mu} g\left(\Lambda_{r}(\lambda) x\right)=g(x)
$$

A vector field $f: \mathbb{R}^{n} \rightarrow \mathbb{R}^{n}$ is said to be $r$-homogeneous of degree $v \in \mathbb{R}\left(\nu \geq-r_{\min }\right)$ if $\forall x \in \mathbb{R}^{n}$ and $\forall \lambda>0$,

$$
f\left(\Lambda_{r}(\lambda) x\right)=\lambda^{v} \Lambda_{r}(\lambda) f(x)
$$

which is equivalent to the $i$-th component of $f$ being a $r$-homogeneous function of degree $r_{i}+v$.

The system (1) is called $r$-homogeneous of degree $v$ if the vector field $f$ is $r$-homogeneous of degree $v$. There are many HOSM systems satisfying Definition $1\left(\right.$ see $\left.^{16}\right)$.

\footnotetext{
${ }^{2}$ It is not a norm in the usual sense, since it does not satisfy the triangle inequality.
} 
Following the results in ${ }^{2,26}$, if the system (1) is $r$-homogeneous of degree $v$ and it admits a unique solution $\chi\left(t, x_{0}\right)$ in forward time with initial condition $x_{0} \in \mathbb{R}^{n}$, then

$$
\Lambda_{r}(\lambda) \chi\left(\lambda^{v} t, x_{0}\right)=\chi\left(t, \Lambda_{r}(\lambda) x_{0}\right)
$$

for all $\lambda>0$ and all $t \geq 0$ where solutions are defined.

By its definition, $\|\cdot\|_{r}$ is a $r$-homogeneous function of degree 1 , and there exists $\underline{\sigma}, \bar{\sigma} \in \mathcal{K}_{\infty}$ such that

$$
\underline{\sigma}\left(\|x\|_{r}\right) \leq\|x\| \leq \bar{\sigma}\left(\|x\|_{r}\right) \quad \forall x \in \mathbb{R}^{n}
$$

i.e. there is a relation between the norms $\|\cdot\|$ and $\|\cdot\|_{r}{ }^{11}$.

Corollary 1. (Efimov et al. ${ }^{11}$ ). Let $r_{\max } \leq 1$ and $\rho \geq 1$, then $\|\cdot\|_{r}$ is locally Lipschitz continuous.

Remark 1. If the system (1) is $r$-homogeneous of degree $\nu$, then it is also $\tilde{r}$-homogeneous for $\tilde{r}=r_{\max }^{-1}\left(r_{1}, \ldots, r_{n}\right)^{T}$ with a degree $\tilde{v}=\frac{v}{r_{\max }}$. Therefore, without loss of generality, it can be always assumed that the Lipschitz continuity of the homogeneous norm $\|\cdot\|_{r}$ (established in Corollary 1 ) is kept with $r_{\max }=1$ and $\rho \geq r_{\max }$ for all homogeneous systems.

\section{2 | Stability}

Following ${ }^{1,27,28}$, the system (1) possessing unique solutions in forward time is called

- Lyapunov stable at the origin if the solution $\chi\left(t, x_{0}\right)$ is defined for all $t \geq 0$ and for each $\epsilon>0$ there exists $\delta>0$ such that for any $\left\|x_{0}\right\|<\delta$, the solution satisfies $\left\|\chi\left(t, x_{0}\right)\right\|<\epsilon$ for all $t \geq 0$.

- (Globally) Asymptotically stable at the origin if it is Lyapunov stable at the origin and, in addition, there exists $\delta_{0}>0$ such that $\lim _{t \rightarrow+\infty}\left\|\chi\left(t, x_{0}\right)\right\|=0$ for each $\left\|x_{0}\right\|<\delta_{0}\left(\delta_{0}\right.$ arbitrarily large $)$.

- (Globally) Finite-time stable at the origin if it is (globally) asymptotically stable and $\left\|\chi\left(t, x_{0}\right)\right\|=0$ for all $t \geq T_{s}\left(x_{0}\right)$ and all $x_{0} \in \mathbb{R}^{n}$, where $T_{s}: \mathbb{R}^{n} \rightarrow \mathbb{R}_{+}$is a settling time function.

For a $V: \mathbb{R} \rightarrow \mathbb{R}_{+}$denote the upper Dini derivative:

$$
\dot{V}^{+}(t)=\limsup _{h \rightarrow 0^{+}} \frac{V(t+h)-V(t)}{h}, \forall t \in \mathbb{R}_{+} .
$$

If $V$ is locally Lipschitz continuous then $\dot{V}^{+}$is finite, and if $V$ is differentiable then $\dot{V}^{+}$is the usual derivative of $V$. For $V: \mathbb{R}^{n} \rightarrow \mathbb{R}_{+}$the generalized directional derivative at $x \in \mathbb{R}^{n}$ in the direction $d \in \mathbb{R}^{n}$ is defined by

$$
D^{+} V(x) d=\limsup _{y \rightarrow x} \frac{V(y+h d)-V(y)}{h} .
$$


For a $r$-homogeneous system (1) of degree $v$, if it is asymptotically stable at origin, then there exists a smooth, $r$-homogeneous, Lyapunov function $V: \mathbb{R}^{n} \rightarrow \mathbb{R}_{+}$of degree $\mu>\max \{0,-v\}$, which satisfies

$$
\begin{aligned}
a\|x\|_{r}^{\mu} \leq V(x) \leq b\|x\|_{r}^{\mu}, & \forall x \in \mathbb{R}^{n}, \\
\sup _{d \in F(x)} D^{+} V(x) d \leq-c V^{1+\frac{v}{\mu}}(x), & \forall x \in \mathbb{R}^{n},
\end{aligned}
$$

for some $0<a \leq b$ and $c>0^{2,26}$. Then, for any $x_{0} \in \mathbb{R}^{n}$,

$$
\left\|\chi\left(t, x_{0}\right)\right\|_{r} \leq \beta\left(\left\|x_{0}\right\|_{r}, t\right) \quad \forall t \geq 0
$$

where

$$
\beta(s, t)= \begin{cases}\left(\left(\frac{b}{a}\right)^{-\frac{v}{\mu}} s^{-v}+\frac{v c a^{\frac{v}{\mu}}}{\mu} t\right)^{-\frac{1}{v}} & t<\frac{-\mu b^{-\frac{v}{\mu}} s^{-v}}{\nu c} \\ 0 & t \geq \frac{-\mu b^{-\frac{v}{\mu}} s^{-v}}{\nu c}\end{cases}
$$

is a $\mathcal{K L}$-function, and it represents a generic parametrization of the upper bound $\beta\left(\left\|x_{0}\right\|_{r}, t\right)$ of $\left\|\chi\left(t, x_{0}\right)\right\|_{r}$ for all $t \geq 0$ in homogeneous systems of negative degree ${ }^{11}$.

Following proposition shows that such a locally Lipschitz continuous and $r$-homogeneous Lyapunov function $V$ possesses a kind of robustness with respect to small deformations under some restrictions.

Proposition 2. (Efimov et al. ${ }^{11}$ ). For a locally Lipschitz continuous and $r$-homogeneous function $V$ of degree $\mu>-v$, assume that the estimates (6) and (7) are satisfied with constants $0<a \leq b$ and $c>0$. Let $\epsilon: \mathbb{R}^{n} \rightarrow \mathbb{R}$ be a locally Lipschitz continuous and $r$-homogeneous function of degree $\mu$, such that, $-a<\underline{\epsilon}$ and $c>\bar{\epsilon}$, where $\underline{\epsilon}=\inf _{y \in S_{r}(1)} \epsilon(y)$ and $\bar{\epsilon}=\sup _{y \in S_{r}(1)} D^{+} \epsilon(y) f(y)$. Then $V^{\prime}(x)=V(x)+\epsilon(x)$ is a locally Lipschitz continuous and $r$-homogeneous Lyapunov function for the system (1).

One important feature of $r$-homogeneous systems is that their rate of convergence is related to their homogeneity degree.

Theorem 1 (Bhat and Bernstein ${ }^{29}$ ). Let the system (1) be $r$-homogeneous of degree $v$, then its origin is a finite-time stable equilibrium point if and only if it is asymptotically stable and the homogeneity degree $v$ is negative.

On the other hand, following the work in ${ }^{11}$ the speed of convergence of the trajectories of $r$-homogeneous systems can be quantified as follows:

Definition 2. Assume the system (1) is asymptotically stable. For $q>1$, define the map $T_{q}: \mathbb{R}^{n} \rightarrow \mathbb{R}_{+}$as the first time the norm of the solution becomes $q$ times smaller than the initial one:

$$
\left\|\chi\left(T_{q}\left(x_{0}\right), x_{0}\right)\right\|_{r}=q^{-1}\left\|x_{0}\right\|_{r}
$$


From its definition, it is easily seen that $T_{q}$ is $r$-homogeneous of degree $-v$ :

$$
T_{q}\left(\Lambda_{r}(\lambda) x_{0}\right)=\lambda^{-\nu} T_{q}\left(x_{0}\right), \quad \forall x_{0} \in \mathbb{R}^{n}, \forall \lambda>0
$$

Definition 3. Assume the system (1) is asymptotically stable. For $q>1$, define a continuous function $\bar{T}_{q}: \mathbb{R}^{n} \rightarrow \mathbb{R}_{+}$as a time from which the solution reaches and stays in the ball with the radius $q$ times smaller than the initial one:

$$
\left\|\chi\left(t, x_{0}\right)\right\|_{r} \leq q^{-1}\left\|x_{0}\right\|_{r}, \quad \forall t \geq \bar{T}_{q}\left(x_{0}\right)
$$

Moreover, define a continuous function $\bar{T}_{q}^{\max }: \mathbb{R}_{+} \rightarrow \mathbb{R}_{+}$as

$$
\bar{T}_{q}^{\max }\left(\left\|x_{0}\right\|_{r}\right)=\sup _{x_{0} \in S_{r}(s)} \bar{T}_{q}\left(x_{0}\right)
$$

Note that $\bar{T}_{q}^{\max }$ is uniform on the sphere $S_{r}(s)$. Hereafter, skipping some ambiguity the function $\bar{T}_{q}^{\text {max }}$ is just represented by $\bar{T}_{q}$ for compactness of notation. Moreover, $\bar{T}_{q}$ is also considered $r$-homogeneous of degree $-v$ :

$$
\bar{T}_{q}\left(\Lambda_{r}(\lambda) x_{0}\right)=\lambda^{-\nu} \bar{T}_{q}\left(x_{0}\right), \quad \forall x_{0} \in \mathbb{R}^{n}, \forall \lambda>0
$$

Taking into account the expression of $\beta$ given in (9), a possible selection for $\bar{T}_{q}$ is

$$
\bar{T}_{q}\left(\left\|x_{0}\right\|_{r}\right)=\frac{\left(\frac{b}{a}\right)^{-\frac{v}{\mu}}-q^{\nu}}{-\frac{\nu}{\mu} c a^{\frac{v}{\mu}}}\left\|x_{0}\right\|_{r}^{-v} \quad v<0 .
$$

\section{4 | CONVERSE LYAPUNOV THEOREMS FOR HOMOGENEOUS DISCONTINUOUS SYSTEMS}

The results of Massera ${ }^{4}$ and Kurzweil ${ }^{3}$ provide the design of a Lyapunov function as the integral of a $\mathcal{K}_{\infty}$ function depending on the system's trajectories, while Persidskii ${ }^{5}$ and Yoshizawa ${ }^{6}$ propose the design of Lyapunov functions based on the supremum of a $\mathcal{K}_{\infty}$ function of the system's solutions. In this section, these classical converse Lyapunov theorems are generalized for $r$ homogeneous discontinuous systems. Also, explicit formulas of $r$-homogeneous and Lipschitz continuous Lyapunov functions are provided. First, let us make some assumptions about the system (1).

Assumption 1. Let the system (1) be $r$-homogeneous of degree $v<0$ and be endowed with the property of uniqueness of Filippov solutions in $\mathbb{R}^{n} \backslash\{0\}$ with no sliding motion on $\mathbb{R}^{n}$ except at the origin. Furthermore, let $f$ be discontinuous only on a set of measure zero $S \subseteq \bigcup_{i=1}^{m} \partial D_{i}$ for disjoint, open and connected sets $D_{i} \subset \mathbb{R}^{n}, i=1, \ldots, m$, where $m>0$ is a finite integer, whose closures cover $\mathbb{R}^{n}$, i.e., $\mathbb{R}^{n}=\bigcup_{i=1}^{m} \bar{D}_{i}$. Moreover, on each $\bar{D}_{i}$ with $i=1, \ldots, m, f$ admits a continuous projection $f_{\mid \bar{D}_{i}}$, which is locally Lipschitz continuous on $D_{i}$, and if $x \in \partial D_{i} \cap \partial D_{j} \subset S$ for $i \neq j \in\{1, \ldots, m\}$, then either $f_{\mid \bar{D}_{i}}(x)$ points into $D_{j}$ or $f_{\mid \bar{D}_{j}}(x)$ points into $D_{i}$. 
Some systems satisfying Assumption 1 are the twisting algorithm, the terminal algorithm ${ }^{3}$ and the quasi-continuous controller, which belong to the family of $\operatorname{HOSM}^{16}$. Under these hypotheses, the solutions of (1) admit a local Lipschitz continuity property with respect to initial conditions on a bounded interval of time:

Lemma 1. Let Assumption 1 be satisfied and the system (1) be globally finite-time stable, then for any $q>1$ there is $L_{S_{r}(1), \bar{T}_{q}(1)}>0$ such that for the solutions of (1)

$$
\left\|\chi\left(t, x_{1}\right)-\chi\left(t, x_{2}\right)\right\| \leq L_{S_{r}(1), \bar{T}_{q}(1)}\left\|x_{1}-x_{2}\right\|
$$

for any $x_{1}, x_{2} \in S_{r}(1)$ and all $t \in\left[0, \bar{T}_{q}(1)\right)$.

Proof. Under Assumption 1, the interval $\left[0, \bar{T}_{q}(1)\right)=\bigcup_{k=0}^{N_{x_{0}}}\left[t_{k}^{x_{0}}, t_{k+1}^{x_{0}}\right)$ for some finite $N_{x_{0}}>0$ for any $x_{0} \in \mathbb{R}^{n}$, where $\chi\left(t_{k}^{x_{0}}, x_{0}\right) \in S$ for all $k \in\left\{0, \ldots, N_{x_{0}}+1\right\}$ (except probably $t_{0}^{x_{0}}$ and $\left.t_{N_{x_{0}}+1}^{x_{0}}\right)$. On each interval $\left(t_{k}^{x_{0}}, t_{k+1}^{x_{0}}\right)$ the trajectory stays in a respective domain $D_{i_{k}}$ with $i_{k} \in\{1, \ldots, m\}$. Since the system is stable and by the definition of $\bar{T}_{q}(1)$,

$$
\chi\left(t, x_{0}\right) \in \mathcal{X}:=\left\{x \in \mathbb{R}^{n}: q^{\prime-1} \leq\|x\|_{r} \leq\left(\frac{b}{a}\right)^{\frac{1}{\mu}}\right\}
$$

for all $t \in\left[0, \bar{T}_{q}(1)\right)$ and $q^{\prime} \in[q,+\infty)$, where $a, b, \mu$ are given in (9). Denote by $L_{i}>0$ the corresponding Lipschitz constant of (1) on $D_{i} \cap \mathcal{X}$ with $i \in\{1, \ldots, m\}, L_{\max }=\max _{i=1, \ldots, m} L_{i}$. In addition, there exist $\theta_{\mathcal{X}}, \Theta_{\mathcal{X}}, L_{\mathcal{X}}>0$ such that

$$
\theta_{\mathcal{X}} \leq\left\|f\left(x_{1}\right)-f\left(x_{2}\right)\right\| \leq L_{\mathcal{X}}\left\|x_{1}-x_{2}\right\|+\Theta_{\mathcal{X}}, \quad \forall x_{1}, x_{2} \in \mathcal{X}
$$

Let us show the Lipschitz property of the solutions of (1) under Assumption 1 for any $x_{1}, x_{2} \in S_{r}(1)$ on the interval $\left[0, \bar{T}_{q}(1)\right.$ ). To this end, denote $\bar{N}=\sup _{x_{0} \in S_{r}(1)} N_{x_{0}}$, which is a finite integer under hypotheses of the lemma.

Consider first the interval $\left[0, t^{\prime}\right)$, where $t^{\prime}=\min \left\{t_{1}^{x_{1}}, t_{1}^{x_{2}}\right\}$ and $\chi\left(t, x_{j}\right) \in D_{i_{j}}$ for $j=1,2$ and $t \in\left(0, t^{\prime}\right)$. If $D_{i_{1}}=D_{i_{2}}$ then using Bellman-Gronwall lemma we obtain:

$$
\begin{aligned}
\left\|\chi\left(t, x_{1}\right)-\chi\left(t, x_{2}\right)\right\| & =\left\|x_{1}-x_{2}+\int_{0}^{t} f\left(\chi\left(s, x_{1}\right)\right)-f\left(\chi\left(s, x_{2}\right)\right) d s\right\| \\
& \leq\left\|x_{1}-x_{2}\right\|+\int_{0}^{t}\left\|f\left(\chi\left(s, x_{1}\right)\right)-f\left(\chi\left(s, x_{2}\right)\right)\right\| d s \\
& \leq\left\|x_{1}-x_{2}\right\|+L_{\max } \int_{0}^{t}\left\|\chi\left(s, x_{1}\right)-\chi\left(s, x_{2}\right)\right\| d s \\
& \leq e^{L_{\max } t}\left\|x_{1}-x_{2}\right\|
\end{aligned}
$$

${ }^{3}$ For a special selection of the controller gains $\beta^{2}>2 \alpha$ the trajectories of the terminal algorithm behave in twisting mode. Therefore, they exhibit sliding mode only at the origin. 
for all $t \in\left[0, t^{\prime}\right)$. If $D_{i_{1}}$ and $D_{i_{2}}$ are distinct sets, then

$$
\begin{aligned}
\left\|\chi\left(t, x_{1}\right)-\chi\left(t, x_{2}\right)\right\| & \leq\left\|x_{1}-x_{2}\right\|+\int_{0}^{t}\left\|f\left(\chi\left(s, x_{1}\right)\right)-f\left(\chi\left(s, x_{2}\right)\right)\right\| d s \\
& \leq\left\|x_{1}-x_{2}\right\|+\int_{0}^{t} L_{\mathcal{X}}\left\|\chi\left(s, x_{1}\right)-\chi\left(s, x_{2}\right)\right\|+\Theta_{\mathcal{X}} d s \\
& \leq\left(1+H_{\mathcal{X}} \bar{T}_{q}(1)\right)\left\|x_{1}-x_{2}\right\|+L_{\mathcal{X}} \int_{0}^{t}\left\|\chi\left(s, x_{1}\right)-\chi\left(s, x_{2}\right)\right\| d s \\
& \leq\left(1+H_{\mathcal{X}} \bar{T}_{q}(1)\right) e^{L_{\mathcal{X}} t}\left\|x_{1}-x_{2}\right\|
\end{aligned}
$$

for all $t \in\left[0, t^{\prime}\right)$ provided that $\Theta_{\mathcal{X}} \leq H_{\mathcal{X}}\left\|x_{1}-x_{2}\right\|$ for some $H_{\mathcal{X}}>0$. Or alternatively,

$$
\begin{aligned}
\left\|\chi\left(t, x_{1}\right)-\chi\left(t, x_{2}\right)\right\| & \leq\left\|x_{1}-x_{2}\right\|+\int_{0}^{t} L_{\mathcal{X}}\left\|\chi\left(s, x_{1}\right)-\chi\left(s, x_{2}\right)\right\|+\Theta_{\mathcal{X}} d s \\
& \leq\left\|x_{1}-x_{2}\right\|+\Theta_{\mathcal{X}} t+L_{\mathcal{X}} \int_{0}^{t}\left\|\chi\left(s, x_{1}\right)-\chi\left(s, x_{2}\right)\right\| d s \\
& \leq\left(1+K_{\mathcal{X}}\right) e^{L_{\mathcal{X}} t}\left\|x_{1}-x_{2}\right\|
\end{aligned}
$$

for all $t \in\left[0, t^{\prime}\right)$ provided that $\Theta_{\mathcal{X}} t \leq K_{\mathcal{X}}\left\|x_{1}-x_{2}\right\|$ for some $K_{\mathcal{X}}>0$. To complement these cases, it remains to consider the scenario with $\Theta_{\mathcal{X}}>H_{\mathcal{X}}\left\|x_{1}-x_{2}\right\|$ and $\Theta_{\mathcal{X}} t>K_{\mathcal{X}}\left\|x_{1}-x_{2}\right\|$, then for a sufficiently big value of $H_{\mathcal{X}}$, the initial conditions belong to a neighborhood of $S$ (since $\left\|x_{1}-x_{2}\right\|<\frac{\Theta_{\mathcal{X}}}{H_{\mathcal{X}}}$ and $x_{1} \in D_{i_{1}}, x_{2} \in D_{i_{2}}$ ). Recalling Assumption 1, in such a case either $\chi\left(t^{\prime}, x_{1}\right) \in D_{i_{2}}$ or $\chi\left(t^{\prime}, x_{2}\right) \in D_{i_{1}}$, hence, there is $S_{\mathcal{X}}>0$ such that $t^{\prime} \leq \frac{S_{\mathcal{X}}}{\theta_{\mathcal{X}}}\left\|x_{1}-x_{2}\right\|$ and for $K_{\mathcal{X}} \geq S_{\mathcal{X}} \frac{\Theta_{\mathcal{X}}}{\theta_{\mathcal{X}}}$ we get a contradiction. Therefore,

$$
\left\|\chi\left(t, x_{1}\right)-\chi\left(t, x_{2}\right)\right\| \leq \mathcal{L}\left\|x_{1}-x_{2}\right\|
$$

for all $t \in\left[0, t^{\prime}\right)$, where

$$
\mathcal{L}=\max \left\{e^{L_{\max } \bar{T}_{q}(1)},\left(1+H_{\mathcal{X}} \bar{T}_{q}(1)\right) e^{L_{\mathcal{X}} \bar{T}_{q}(1)},\left(1+K_{\mathcal{X}}\right) e^{L_{\mathcal{X}} \bar{T}_{q}(1)}\right\}
$$

Now, consider $t \in\left[t^{\prime}, t^{\prime \prime}\right)$ for $t^{\prime \prime}=\min \left\{\max \left\{t_{1}^{x_{1}}, t_{1}^{x_{2}}\right\}, \min \left\{t_{2}^{x_{1}}, t_{2}^{x_{2}}\right\}\right\}$, and applying the same argumentation as previously:

$$
\left\|\chi\left(t, \chi\left(t^{\prime}, x_{1}\right)\right)-\chi\left(t, \chi\left(t^{\prime}, x_{2}\right)\right)\right\| \leq \mathcal{L}\left\|\chi\left(t^{\prime}, x_{1}\right)-\chi\left(t^{\prime}, x_{2}\right)\right\| \leq \mathcal{L}^{2}\left\|x_{1}-x_{2}\right\|
$$

for all $t \in\left[t^{\prime}, t^{\prime \prime}\right)$, and we used continuity of the solutions for the time instant $t^{\prime}$. Therefore,

$$
\left\|\chi\left(t, x_{1}\right)-\chi\left(t, x_{2}\right)\right\| \leq \max \left\{\mathcal{L}, \mathcal{L}^{2}\right\}\left\|x_{1}-x_{2}\right\|
$$


for all $t \in\left[0, t^{\prime \prime}\right)$. The same consideration can be repeated for all other intervals of time in a similar way leading to the estimate:

$$
\left\|\chi\left(t, x_{1}\right)-\chi\left(t, x_{2}\right)\right\| \leq \max _{j=0, \ldots, 2 \bar{N}} \mathcal{L}^{j}\left\|x_{1}-x_{2}\right\|
$$

for all $t \in\left[0, \bar{T}_{q}(1)\right)$, and the desired result follows for $L_{S_{r}(1), \bar{T}_{q}(1)}=\max \left\{\mathcal{L}, \mathcal{L}^{2 \bar{N}}\right\}$.

\section{1 | Design based on the integral of the system's trajectories}

Following the work in ${ }^{11}$, where the results of Massera ${ }^{4}$ and Kurzweil ${ }^{3}$ were extended to $r$-homogeneous systems of positive degree, the next theorem presents an extension of such results for systems satisfying Assumption 1.

Theorem 2. Let the system (1) satisfy Assumption 1. Then the following is equivalent:

(i) The discontinuous system (1) is globally finite-time stable at the origin.

(ii) For all $x \in \mathbb{R}^{n}$ and $\mu \geq 1$, there exists $q>1$ such that the function

$$
U(x)=\int_{0}^{T_{q}^{\max }\left(\|x\|_{r}\right)}\|\chi(t, x)\|_{r}^{\mu} d t,
$$

where $T_{q}^{\max }(s)=\sup _{x \in S_{r}(s)} T_{q}(x)$ and $T_{q}$ is given by Definition 2, is locally Lipschitz continuous on $\mathbb{R}^{n} \backslash\{0\}$, $r$-homogeneous of degree $\mu-v$, and satisfies

$$
\begin{gathered}
T_{q}^{\min } q^{-\mu}\|x\|_{r}^{\mu-\nu} \leq U(x) \leq T_{q}^{\max }(1) \frac{b}{a}\|x\|_{r}^{\mu-\nu}, \forall x \in \mathbb{R}^{n}, \\
D^{+} U(x) F(x)<0, \text { for a.a. } x \in \mathbb{R}^{n} \backslash\{0\},
\end{gathered}
$$

where $T_{q}^{\min }=\inf _{x \in S_{r}(1)} T_{q}(x)$.

Proof. The existence of the Lyapunov function (11) satisfying the statement of Theorem 2 under a negative homogeneity degree $v$ is enough to conclude the finite-time stability of the origin of the discontinuous system (1). Conversely, assuming the first claim in Theorem 2, the existence of the Lyapunov function (11) is proven as follows.

Assuming stability for the system (1), by definition of $T_{q}$ and the inequality (8), with the function $\beta$ is given by (9), the solution $\chi\left(t, x_{0}\right)$ is bounded for all $t \in\left[0, T_{q}^{\max }\left(\|x\|_{r}\right)\right]$ and for any $x_{0} \in S_{r}(1)$. Thus, $q^{\prime}$, with $q^{\prime}>q>1$, can be selected so that

$$
\left\|\chi\left(t, x_{0}\right)\right\|_{r} \in\left[\frac{1}{q^{\prime}},\left(\frac{b}{a}\right)^{\frac{1}{\mu}}\right] .
$$

For any $x_{0} \in \mathbb{R}^{n}$ define

$$
U\left(x_{0}\right)=\int_{0}^{T_{q}^{\max }\left(\left\|x_{0}\right\|_{r}\right)}\left\|\chi\left(t, x_{0}\right)\right\|_{r}^{\mu} d t,
$$


where for $q>1, T_{q}^{\max }\left(\left\|x_{0}\right\|_{r}\right)$ is defined in Theorem 2. The function (13) is $r$-homogeneous of degree $\mu-v$ since for all $x_{0} \in \mathbb{R}^{n}$ and $\lambda>0$

$$
\begin{aligned}
U\left(\Lambda_{r}(\lambda) x_{0}\right) & =\int_{0}^{T_{q}^{\max }\left(\left\|\Lambda_{r}(\lambda) x_{0}\right\|_{r}\right)}\left\|\chi\left(t, \Lambda_{r}(\lambda) x_{0}\right)\right\|_{r}^{\mu} d t \\
& =\int_{0}^{\lambda^{-v} T_{q}^{\max }\left(\left\|x_{0}\right\|_{r}\right)}\left\|\Lambda_{r}(\lambda) \chi\left(\lambda^{\nu} t, x_{0}\right)\right\|_{r}^{\mu} d t \\
& =\lambda^{\mu-\nu} \int_{0}^{T_{q}^{\max }\left(\left\|x_{0}\right\|_{r}\right)}\left\|\chi\left(\tau, x_{0}\right)\right\|_{r}^{\mu} d \tau=\lambda^{\mu-\nu} U\left(x_{0}\right),
\end{aligned}
$$

where the change of variables $\tau=\lambda^{v} t$ was used. By construction $U(0)=0$. Moreover, considering (12) and for any $x_{0} \in S_{r}(1)$

$$
U\left(x_{0}\right)=\int_{0}^{T_{q}^{\max }(1)}\left\|\chi\left(t, x_{0}\right)\right\|_{r}^{\mu} d t \geq \int_{0}^{T_{q}\left(x_{0}\right)} q^{-\mu} d t=T_{q}\left(x_{0}\right) q^{-\mu},
$$

and

$$
U\left(x_{0}\right)=\int_{0}^{T_{q}^{\max }(1)}\left\|\chi\left(t, x_{0}\right)\right\|_{r}^{\mu} d t \leq \int_{0}^{T_{q}^{\max }(1)} \frac{b}{a} d t=T_{q}^{\max }(1) \frac{b}{a} .
$$

Hence, by homogeneity

$$
T_{q}^{\min } q^{-\mu}\|x\|_{r}^{\mu-\nu} \leq U(x) \leq T_{q}^{\max }(1) \frac{b}{a}\|x\|_{r}^{\mu-\nu}
$$

for all $x \in \mathbb{R}^{n}$, where $T_{q}^{\min }$ and $T_{q}^{\max }(1)$ are defined in Theorem 2 . In order to analyze the Lipschitz continuity of the function (13), consider two states $x_{1}, x_{2} \in S_{r}(1)$ and

$$
\begin{aligned}
\left|U\left(x_{1}\right)-U\left(x_{2}\right)\right| & =\left|\int_{0}^{T_{q}^{\max }\left(\left\|x_{1}\right\|_{r}\right)}\left\|\chi\left(t, x_{1}\right)\right\|_{r}^{\mu} d t-\int_{0}^{T_{q}^{\max }\left(\left\|x_{2}\right\|_{r}\right)}\left\|\chi\left(t, x_{2}\right)\right\|_{r}^{\mu} d t\right| \\
& =\left|\int_{0}^{T_{q}^{\max }(1)}\left\|\chi\left(t, x_{1}\right)\right\|_{r}^{\mu}-\left\|\chi\left(t, x_{2}\right)\right\|_{r}^{\mu} d t\right| \\
& \leq \int_{0}^{T_{q}^{\max }(1)}\left|\left\|\chi\left(t, x_{1}\right)\right\|_{r}^{\mu}-\left\|\chi\left(t, x_{2}\right)\right\|_{r}^{\mu}\right| d t
\end{aligned}
$$

Under Assumption 1, the Lipschitz continuity of the trajectories $\chi\left(t, x_{0}\right)$ w.r.t. $x_{0}$ is established by Lemma 1 for all $t \in\left[0, \bar{T}_{q}(1)\right]$ (note that $\left.T_{q}^{\max }(1) \leq \bar{T}_{q}(1)\right)$. Recall, from inequality (12) the solutions $\chi\left(t, x_{0}\right)$ are all bounded for any $x_{0} \in S_{r}(1)$ and all $t \in\left[0, T_{q}^{\max }(1)\right]$. Moreover, the Lipschitz continuity of the $r$-homogeneous norm is established by Corollary 1 and the power function $x \mapsto x^{\mu}$ is Lipschitz continuous for any $\mu>1$ as well. As the composition of Lipschitz continuous functions inherits the property, it is obtained

$$
\left|\left\|\chi\left(t, x_{1}\right)\right\|_{r}^{\mu}-\left\|\chi\left(t, x_{2}\right)\right\|_{r}^{\mu}\right| \leq \bar{L}\left\|x_{1}-x_{2}\right\|,
$$


for all $t \in\left[0, T_{q}^{\max }(1)\right]$, all $x_{1}, x_{2} \in S_{r}(1)$ and some $\bar{L}>0$. Consequently, substituting (16) in (15)

$$
\left|U\left(x_{1}\right)-U\left(x_{2}\right)\right| \leq \int_{0}^{T_{q}^{\max }(1)} \bar{L}\left\|x_{1}-x_{2}\right\| d t \leq T_{q}^{\max } \bar{L}\left\|x_{1}-x_{2}\right\|
$$

for all $x_{1}, x_{2} \in S_{r}(1)$, then the function $U$ is locally Lipschitz continuous on the unit sphere, and by homogeneity, it is Lipschitz continuous in $\mathbb{R}^{n} \backslash\{0\}$ as needed.

Along a trajectory $\chi\left(t, x_{0}\right)$, the derivative of $U\left(\chi\left(t, x_{0}\right)\right)$ at the point $x_{0}$ is given by

$$
D^{+} U\left(x_{0}\right) F\left(x_{0}\right)=\limsup _{h \rightarrow 0^{+}} \frac{1}{h}\left[U\left(\chi\left(h, x_{0}\right)\right)-U\left(x_{0}\right)\right] .
$$

So, it is enough to show that the function (13) is decreasing along any trajectory of the system (1), i.e., $U\left(\chi\left(t, x_{0}\right)\right)<U\left(x_{0}\right)$, for any (small) $t \in\left(0, T_{q}^{\max }\left(\left\|x_{0}\right\|_{r}\right)\right)$, to conclude that $D^{+} U\left(x_{0}\right) F\left(x_{0}\right)<0$ for almost all $x_{0} \in \mathbb{R}^{n} \backslash\{0\}$. From the semi-group property we have that $\left\|\chi\left(\tau, \chi\left(t, x_{0}\right)\right)\right\|_{r}=\left\|\chi\left(\tau+t, x_{0}\right)\right\|_{r}$, for all $t, \tau \in \mathbb{R}_{+}$and $x_{0} \in \mathbb{R}^{n}$. Thus, for any $x_{0} \in \mathbb{R}^{n}$ and all $t \in \mathbb{R}_{+}$

$$
\begin{aligned}
U\left(\chi\left(t, x_{0}\right)\right) & =\int_{0}^{T_{q}^{\max }\left(\left\|\chi\left(t, x_{0}\right)\right\|_{r}\right)}\left\|\chi\left(\tau, \chi\left(t, x_{0}\right)\right)\right\|_{r}^{\mu} d \tau \\
& =\int_{0}^{T_{q}^{\max }\left(\left\|\chi\left(t, x_{0}\right)\right\|_{r}\right)}\left\|\chi\left(\tau+t, x_{0}\right)\right\|_{r}^{\mu} d \tau \\
& =\int_{0}^{t+T_{q}^{\max }\left(\left\|\chi\left(t, x_{0}\right)\right\|_{r}\right)}\left\|\chi\left(s, x_{0}\right)\right\|_{r}^{\mu} d s-\int_{0}^{t}\left\|\chi\left(s, x_{0}\right)\right\|_{r}^{\mu} d s \\
& =\int_{t+T_{q}^{\max }\left(\left\|\chi\left(t, x_{0}\right)\right\|_{r}\right)}^{t}\left\|\chi\left(s, x_{0}\right)\right\|_{r}^{\mu} d s-\int_{0}^{T_{q}^{\max }\left(\left\|x_{0}\right\|_{r}\right)}\left\|\chi\left(s, x_{0}\right)\right\|_{r}^{\mu} d s+\int_{0}^{T_{q}^{\max }\left(\left\|x_{0}\right\|_{r}\right)}\left\|\chi\left(s, x_{0}\right)\right\|_{r}^{\mu} d s .
\end{aligned}
$$

with $q>1$ and $\mu>1$. The first term in the latter expression depends on $q$, the second one is negative and the third one corresponds to the Lyapunov function (19). Hence, let us show that growing $q$ warranty the decreasing of the Lyapunov function (19) along any trajectory $\chi\left(t, x_{0}\right)$. So, in the first integral

$$
\left\|\chi\left(s, x_{0}\right)\right\|_{r} \leq \beta\left(x_{0}, T_{q}^{\max }\left(\left\|x_{0}\right\|_{r}\right)\right) \quad \forall s \geq T_{q}^{\max }\left(\left\|x_{0}\right\|_{r}\right)
$$

where $\beta$ is a $\mathcal{K} \mathcal{L}$-function given by (9). Note that $T_{q}^{\max }\left(\left\|x_{0}\right\|_{r}\right)$ grows with $q$ and $\beta\left(x_{0}, T_{q}^{\max }\left(\left\|x_{0}\right\|_{r}\right)\right) \rightarrow 0$ as $q \rightarrow \infty$. For the second integral, since the function $\left\|\chi\left(t, x_{0}\right)\right\|_{r}^{\mu}$ is continuous w.r.t. $t$, for any $\epsilon>0$ there exist $t_{1}>0$, such that, if $0 \leq t<t_{1}$ then

$$
\left|\left\|\chi\left(t_{1}, x_{0}\right)\right\|_{r}^{\mu}-\left\|\chi\left(0, x_{0}\right)\right\|_{r}^{\mu}\right|<\epsilon
$$

and

$$
\left\|x_{0}\right\|_{r}-\epsilon<\left\|\chi\left(t, x_{0}\right)\right\|_{r}<\left\|x_{0}\right\|_{r}+\epsilon .
$$


Thus, for $t \in\left[0, t_{1}\right)$, it is derived

$$
\begin{aligned}
U\left(\chi\left(t, x_{0}\right)\right)-U\left(x_{0}\right) & \leq \int_{T_{q}^{\max }\left(\left\|x_{0}\right\|_{r}\right)}^{t+T_{q}^{\max }\left(\left\|\chi\left(t, x_{0}\right)\right\|_{r}\right)} \beta^{\mu}\left(x_{0}, T_{q}^{\max }\left(\left\|x_{0}\right\|_{r}\right)\right) d s-\int_{0}^{t}\left(\left\|x_{0}\right\|_{r}-\epsilon\right)^{\mu} d s \\
& \leq \beta^{\mu}\left(x_{0}, T_{q}^{\max }\left(\left\|x_{0}\right\|_{r}\right)\right)\left(t+T_{q}^{\max }\left(\left\|\chi\left(t, x_{0}\right)\right\|_{r}\right)-T_{q}^{\max }\left(\left\|x_{0}\right\|_{r}\right)\right)-\left(\left\|x_{0}\right\|_{r}-\epsilon\right)^{\mu} t .
\end{aligned}
$$

By the homogeneity of $T_{q}^{\max }$ :

$$
T_{q}^{\max }\left(\left\|\chi\left(t, x_{0}\right)\right\|_{r}\right)=c(t) T_{q}^{\max }\left(\left\|x_{0}\right\|_{r}\right)
$$

where $c(t)=\left(\frac{\left\|\chi\left(t, x_{0}\right)\right\|_{r}}{\left\|x_{0}\right\|_{r}}\right)^{-v}$. Let $t \in\left[0, t_{1}\right)$, then

$$
\begin{aligned}
U\left(\chi\left(t, x_{0}\right)\right)-U\left(x_{0}\right) & \leq \beta^{\mu}\left(x_{0}, T_{q}^{\max }\left(\left\|x_{0}\right\|_{r}\right)\right)\left(t+(c(t)-1) T_{q}^{\max }\left(\left\|x_{0}\right\|_{r}\right)\right)-\left(\left\|x_{0}\right\|_{r}-\epsilon\right)^{\mu} t \\
& \leq-\left(\left(\left\|x_{0}\right\|_{r}-\epsilon\right)^{\mu}-\beta^{\mu}\left(x_{0}, T_{q}^{\max }\left(\left\|x_{0}\right\|_{r}\right)\right)\right) t+|c(t)-1| \beta^{\mu}\left(x_{0}, T_{q}^{\max }\left(\left\|x_{0}\right\|_{r}\right)\right) T_{q}^{\max }\left(\left\|x_{0}\right\|_{r}\right)
\end{aligned}
$$

Let us consider the behavior of $\mathrm{U}$ for $t \in\left[0, t_{1}\right]$ and $x_{0} \in S_{r}(1)$. Since $-1<v<0$, if $\left\|\chi\left(t, x_{0}\right)\right\|_{r}>1$, for these small instants of time, then $\left\|\chi\left(t, x_{0}\right)\right\|_{r}^{-v}<\left\|\chi\left(t, x_{0}\right)\right\|_{r}$, on the contrary, if $\left\|\chi\left(t, x_{0}\right)\right\|_{r}<1$, then $\left\|\chi\left(t, x_{0}\right)\right\|_{r}^{-v}>\left\|\chi\left(t, x_{0}\right)\right\|_{r}$, hence, from both cases it can be concluded that

$$
\left|\left\|\chi\left(t, x_{0}\right)\right\|_{r}^{-v}-1\right| \leq\left|\left\|\chi\left(t, x_{0}\right)\right\|_{r}-1\right|
$$

and by homogeneity the property hold for all $x_{0} \in \mathbb{R}^{n}$ and $t \in\left[0, t_{1}\right]$, such that,

$$
c(t)-1=\left(\frac{\left\|\chi\left(t, x_{0}\right)\right\|_{r}}{\left\|x_{0}\right\|_{r}}\right)^{-\nu}-1 \leq \frac{\left\|\chi\left(t, x_{0}\right)\right\|_{r}}{\left\|x_{0}\right\|_{r}}-1,
$$

where the fact that $v \in[-1,0)$ since $r_{\max }=1$ was used for Lipschitz continuity of $\|\cdot\|_{r}$, and denote the respective Lipschitz constant by $L_{\|\cdot\|_{r}, x_{0}}^{-v}>0$ for the set where the trajectories behave. Applying the last observation

$$
c(t)-1 \leq L_{\|\cdot\|_{r}, x_{0}}^{-v} \frac{\left\|\chi\left(t, x_{0}\right)-x_{0}\right\|}{\left\|x_{0}\right\|_{r}},
$$

Since $\chi\left(t, x_{0}\right)=x_{0}+\int_{0}^{t} f\left(\chi\left(\tau, x_{0}\right)\right) d \tau$, and $f$ is bounded, then

$$
\left\|\chi\left(t, x_{0}\right)-x_{0}\right\| \leq \int_{0}^{t}\left\|f\left(\chi\left(s, x_{0}\right)\right)\right\| d s \leq f_{\max , x_{0}} t,
$$

where $f_{\max , x_{0}}=\sup _{t \geq 0}\left\|f\left(\chi\left(t, x_{0}\right)\right)\right\|$. Thus,

$$
\begin{aligned}
U\left(\chi\left(t, x_{0}\right)\right)-U\left(x_{0}\right) & \leq-\left[\left(\left\|x_{0}\right\|_{r}-\epsilon\right)^{\mu}-\beta^{\mu}\left(x_{0}, T_{q}^{\max }\left(\left\|x_{0}\right\|_{r}\right)\right)-L_{\|\cdot\|_{r}, x_{0}} \frac{f_{\max , x_{0}}}{\left\|x_{0}\right\|_{r}} \beta^{\mu}\left(x_{0}, T_{q}^{\max }\left(\left\|x_{0}\right\|_{r}\right)\right) T_{q}^{\max }\left(\left\|x_{0}\right\|_{r}\right)\right] t \\
& \leq-\left[\left(\left\|x_{0}\right\|_{r}-\epsilon\right)^{\mu}-\kappa_{x_{0}, q} \beta^{\mu}\left(x_{0}, T_{q}^{\max }\left(\left\|x_{0}\right\|_{r}\right)\right)\right] t
\end{aligned}
$$

for $t \in\left[0, t_{1}\right]$, where $\kappa_{x_{0}, q}=1+L_{\|\cdot\|_{r}, x_{0}} \frac{f_{\max , x_{0}}}{\left\|x_{0}\right\|_{r}} T_{q}^{\max }\left(\left\|x_{0}\right\|_{r}\right)$. Note that the first term in brackets of the right-hand side of the latter expression is negative if $\epsilon<\left\|x_{0}\right\|_{r}$ is selected, and the second one decreases monotonically to zero with growing $q$, 
and $\kappa_{x_{0}, q}$ stays bounded due to the system (1) is assumed finite-time stable and $T_{q}^{\max }(s)<T_{s}(s)$ where $T_{s}$ is the settling time function. This implies that there is a (finite) value $q^{*}$ such that for $q>q^{*}$ the inequality $U(\chi(t, x))-U(x)<0$ holds. Due to homogeneity, it is sufficient to verify this only on the unit sphere $S_{r}(1)$ and since it is a compact set the existence of a constant value $q^{*}>1$ is concluded. Consequently, $U$ is decreasing along any trajectories of the system (1) for almost all $x_{0} \in \mathbb{R}^{n}$. Finally, this completes the proof of Theorem 2.

\section{2 | Design based on the supremum of the system's trajectories}

The following theorem presents an explicit formula of a Lyapunov function based on the supremum of the trajectories. It extends the converse theorems of Persidskii ${ }^{5}$ and Yoshizawa ${ }^{6}$ to discontinuous and $r$-homogeneous systems of negative degree.

First, let $\varrho>0$ be a real parameter satisfying $|\varrho \nu|>1$. Define a function $k: \mathbb{R}_{+} \times \mathbb{R}^{n} \rightarrow \mathbb{R}_{+}$by

$$
k(t, x)=\frac{\|\chi(t, x)\|_{r}^{\rho v} t^{o}+\kappa_{1}}{\|\chi(t, x)\|_{r}^{\rho v} t^{o}+\kappa_{2}},
$$

for given parameters $0<\kappa_{1}<\kappa_{2}<+\infty$.

Theorem 3. Let the system (1) satisfy Assumption 1. Then, the following claims are equivalent:

(i) The discontinuous system (1) is globally finite-time stable at the origin.

(ii) For all $x \in \mathbb{R}^{n}$, there exists $q>1$ such that

$$
V(x)=\sup _{t \in\left[0, T_{q}(x)\right]}\left\{\|\chi(t, x)\|_{r} k(t, x)\right\}
$$

where $T_{q}$ is given in Definition 2, is a locally Lipschitz continuous on $\mathbb{R}^{n} \backslash\{0\}$ and $\mathbf{r}$-homogeneous function of degree 1 satisfying

$$
\begin{gathered}
\frac{\kappa_{1}}{\kappa_{2}}\|x\|_{r} \leq V(x) \leq\left(\frac{b}{a}\right)^{\frac{1}{\mu}}\|x\|_{r} \text { for all } x \in \mathbb{R}^{n}, \\
D^{+} V(x) f(x)<0 \quad \text { for a.a. } x \in \mathbb{R}^{n} \backslash\{0\}
\end{gathered}
$$

Proof. The existence of the Lyapunov function (19) satisfying the statement of Theorem 3 under a negative homogeneity degree $v$ is enough to conclude the finite-time stability of the origin of the discontinuous system (1). Conversely, assuming the first claim in Theorem 3 the existence of Lyapunov function (19) is proven as follows.

First, recall that $v<0$ and the system (1) is assumed to be finite-time stable. The trajectories satisfy inequality (8), with a function $\beta$ being decreasing in time, e.g., the one given by (9). Therefore, there exists $T_{x_{0}}$ such that $\left\|\chi\left(t, x_{0}\right)\right\|_{r}=0$ for all 
$t \geq T_{x_{0}}$. Thus, the supremum of the function $\left\|\chi\left(t, x_{0}\right)\right\|_{r}$ arises on the interval of time $t \in\left[0, T_{x_{0}}\right]$, i.e.,

$$
\sup _{t \in\left[0, T_{x_{0}}\right]}\left\{\left\|\chi\left(\tau, x_{0}\right)\right\|_{r}\right\}=\sup _{t \geq 0}\left\{\left\|\chi\left(t, x_{0}\right)\right\|_{r}\right\} .
$$

Further, note that function $\kappa$ defined in (18) is lower and upper bounded by $\frac{\kappa_{1}}{\kappa_{2}} \leq|k(t, \cdot)|<1$ and $V\left(x_{0}\right) \geq \frac{\kappa_{1}}{\kappa_{2}}\left\|x_{0}\right\|_{r}$ for all $0 \leq t<T_{x_{0}}$. Recalling Definitions 2 and 3, there exists the time $\bar{T}_{\frac{\kappa_{2}}{\kappa_{1}}}\left(x_{0}\right)$, such that,

$$
\left\|\chi\left(t, x_{0}\right)\right\|_{r} \leq \frac{\kappa_{1}}{\kappa_{2}}\left\|x_{0}\right\|_{r}, \quad \forall t \geq \bar{T}_{\frac{\kappa_{2}}{\kappa_{1}}}\left(x_{0}\right)
$$

and for $q>1$ sufficiently large, there exist also the time $T_{q}\left(x_{0}\right) \geq \bar{T}_{\frac{\kappa_{2}}{\kappa_{1}}}\left(x_{0}\right)$ when the trajectories $\chi\left(t, x_{0}\right)$ reach the sphere of radius $\frac{1}{q}\left\|x_{0}\right\|_{r}$ for the first time and they never leave the ball of radius $\frac{\kappa_{1}}{\kappa_{2}}\left\|x_{0}\right\|_{r}$, thereafter. Consequently,

$$
V\left(x_{0}\right)=\sup _{t \in\left[0, T_{q}\left(x_{0}\right)\right]}\left\{\left\|\chi\left(t, x_{0}\right)\right\|_{r} k\left(t, x_{0}\right)\right\}=\sup _{t \geq 0}\left\{\left\|\chi\left(t, x_{0}\right)\right\|_{r} k\left(t, x_{0}\right)\right\}
$$

for such a choice of $q$. In the sequel, both expressions for $V\left(x_{0}\right)$ will be used indistinctly.

So, considering equation (8) (see function $\beta$ in (9)) and the facts $\chi\left(0, x_{0}\right)=x_{0}$ and $\frac{\kappa_{1}}{\kappa_{2}} \leq|k(t, \cdot)|<1$, the function $(20)$ satisfies $V(0)=0$ (since $x=0$ is an equilibrium point), and

$$
\frac{\kappa_{1}}{\kappa_{2}}\left\|x_{0}\right\|_{r} \leq V\left(x_{0}\right) \leq\left(\frac{b}{a}\right)^{\frac{1}{\mu}}\left\|x_{0}\right\|_{r} \text { for all } x_{0} \in \mathbb{R}^{n}
$$

Now, since the system (1) is $r$-homogeneous of degree $v<0$ and the norm $\|\cdot\|_{r}$ is $r$-homogeneous of degree 1 then

$$
\left\|\chi\left(t, \Lambda_{r}(\lambda) x_{0}\right)\right\|_{r}=\left\|\Lambda_{r}(\lambda) \chi\left(\lambda^{v} t, x_{0}\right)\right\|_{r}=\lambda\left\|\chi\left(\lambda^{v} t, x_{0}\right)\right\|_{r}
$$

Also,

$$
k\left(t, \Lambda_{r}(\lambda) x_{0}\right)=\frac{\left\|\chi\left(t, \Lambda_{r}(\lambda) x_{0}\right)\right\|_{r}^{\rho v} t^{\rho}+\kappa_{1}}{\left\|\chi\left(t, \Lambda_{r}(\lambda) x_{0}\right)\right\|_{r}^{\rho v} t^{\rho}+\kappa_{2}}=\frac{\left\|\chi\left(\lambda^{v} t, x_{0}\right)\right\|_{r}^{\rho v} \lambda^{\rho v} t^{\rho}+\kappa_{1}}{\left\|\chi\left(\lambda^{v} t, x_{0}\right)\right\|_{r}^{\rho v} \lambda^{\rho v} t^{\rho}+\kappa_{2}}=k\left(\lambda^{v} t, x_{0}\right)
$$

for all $x_{0} \in \mathbb{R}^{n}, t \in \mathbb{R}_{+}$. These properties imply that for all $x_{0} \in \mathbb{R}^{n}$ and $\lambda>0$ :

$$
\begin{aligned}
V\left(\Lambda_{r}(\lambda) x_{0}\right) & =\sup _{t \geq 0}\left\{\left\|\chi\left(t, \Lambda_{r}(\lambda) x_{0}\right)\right\|_{r} k\left(t, \Lambda_{r}(\lambda) x_{0}\right)\right\} \\
& =\lambda \sup _{s \geq 0}\left\{\left\|\chi\left(s, x_{0}\right)\right\|_{r} k\left(s, x_{0}\right)\right\}=\lambda V\left(x_{0}\right)
\end{aligned}
$$

where the substitution $s=\lambda^{v} t$ was used, then $V$ is $r$-homogeneous of degree 1 . Now, to prove the Lipschitz continuity of $V$ consider $x_{1}, x_{2} \in S_{r}(1)$ and $T_{q}^{\max }(1)=\sup _{x_{0} \in S_{r}(1)} T_{q}\left(x_{0}\right)$, such that,

$$
\left|V\left(x_{1}\right)-V\left(x_{2}\right)\right|=\left|\sup _{t \in\left[0, T_{q}^{\max }(1)\right]}\left\{\left\|\chi\left(t, x_{1}\right)\right\|_{r} k\left(t, x_{1}\right)\right\}-\sup _{t \in\left[0, T_{q}^{\max }(1)\right]}\left\{\left\|\chi\left(t, x_{2}\right)\right\|_{r} k\left(t, x_{2}\right)\right\}\right| .
$$


Adding a zero: $\sup _{t \in\left[0, T_{q}^{\max (1)]}\right.}\left\{\left\|\chi\left(t, x_{2}\right)\right\|_{r} k\left(t, x_{1}\right)\right\}-\sup _{t \in\left[0, T_{q}^{\max }(1)\right]}\left\{\left\|\chi\left(t, x_{2}\right)\right\|_{r} k\left(t, x_{1}\right)\right\}=0$, and applying the triangle inequality for the absolute value,

$$
\begin{aligned}
\left|V\left(x_{1}\right)-V\left(x_{2}\right)\right| \leq\left|\sup _{t \in\left[0, T_{q}^{\max }(1)\right]}\left\{\left\|\chi\left(t, x_{1}\right)\right\|_{r} k\left(t, x_{1}\right)\right\}-\sup _{t \in\left[0, T_{q}^{\max }(1)\right]}\left\{\left\|\chi\left(t, x_{2}\right)\right\|_{r} k\left(t, x_{1}\right)\right\}\right| \\
+\left|\sup _{t \in\left[0, T_{q}^{\max }(1)\right]}\left\{\left\|\chi\left(t, x_{2}\right)\right\|_{r} k\left(t, x_{2}\right)\right\}-\sup _{t \in\left[0, T_{q}^{\max }(1)\right]}\left\{\left\|\chi\left(t, x_{2}\right)\right\|_{r} k\left(t, x_{1}\right)\right\}\right|,
\end{aligned}
$$

and since the absolute value of difference of supremums is less than the supremum of absolute value of difference, then

$$
\sup _{t \in\left[0, T_{q}^{\max (1)]}\right.}\left\{\left|\left\|\chi\left(t, x_{1}\right)\right\|_{r}-\left\|\chi\left(t, x_{2}\right)\right\|_{r}\right| k\left(t, x_{1}\right)\right\} \geq\left|\sup _{t \in\left[0, T_{q}^{\max }(1)\right]}\left\{\left\|\chi\left(t, x_{1}\right)\right\|_{r} k\left(t, x_{1}\right)\right\}-\sup _{t \in\left[0, T_{q}^{\max }(1)\right]}\left\{\left\|\chi\left(t, x_{2}\right)\right\|_{r} k\left(t, x_{1}\right)\right\}\right|,
$$

and

$$
\sup _{t \in\left[0, T_{q}^{\max }(1)\right]}\left\{\left\|\chi\left(t, x_{2}\right)\right\|_{r}\left|k\left(t, x_{2}\right)-k\left(t, x_{1}\right)\right|\right\} \geq\left|\sup _{t \in\left[0, T_{q}^{\max }(1)\right]}\left\{\left\|\chi\left(t, x_{2}\right)\right\|_{r} k\left(t, x_{2}\right)\right\}-\sup _{t \in\left[0, T_{q}^{\max }(1)\right]}\left\{\left\|\chi\left(t, x_{2}\right)\right\|_{r} k\left(t, x_{1}\right)\right\}\right|,
$$

hence it is obtained that

$$
\left|V\left(x_{1}\right)-V\left(x_{2}\right)\right| \leq \sup _{t \in\left[0, T_{q}^{\max }(1)\right]}\left\{\left|\left\|\chi\left(t, x_{1}\right)\right\|_{r}-\left\|\chi\left(t, x_{2}\right)\right\|_{r}\right| k\left(t, x_{1}\right)\right\}+\sup _{t \in\left[0, T_{q}^{\max }(1)\right]}\left\{\left\|\chi\left(t, x_{2}\right)\right\|_{r}\left|k\left(t, x_{2}\right)-k\left(t, x_{1}\right)\right|\right\} .
$$

From (18) it can be seen that $\left|k\left(t, x_{0}\right)\right|<1$, and from (9) $\left\|\chi\left(t, x_{0}\right)\right\|_{r} \leq \frac{b}{a}$, hence

$$
\left|V\left(x_{1}\right)-V\left(x_{2}\right)\right| \leq \sup _{t \in\left[0, T_{q}^{\max (1)]}\right.}\left|\left\|\chi\left(t, x_{1}\right)\right\|_{r}-\left\|\chi\left(t, x_{2}\right)\right\|_{r}\right|+\frac{b}{a} \sup _{t \in\left[0, T_{q}^{\max (1)]}\right.}\left|k\left(t, x_{2}\right)-k\left(t, x_{1}\right)\right| .
$$

Again, from (18),

$$
\left|k\left(t, x_{2}\right)-k\left(t, x_{1}\right)\right|=\frac{\left|\left\|\chi\left(\tau, x_{2}\right)\right\|_{r}^{\rho v}-\left\|\chi\left(\tau, x_{1}\right)\right\|_{r}^{\rho v}\right|\left(\kappa_{2}-\kappa_{1}\right) t^{\rho}}{\left(\left\|\chi\left(\tau, x_{2}\right)\right\|_{r}^{\rho v} t^{\rho}+\kappa_{2}\right)\left(\left\|\chi\left(\tau, x_{1}\right)\right\|_{r}^{\rho v} t^{\rho}+\kappa_{2}\right)}
$$

and

$$
\left|\left\|\chi\left(\tau, x_{2}\right)\right\|_{r}^{\rho v}-\left\|\chi\left(\tau, x_{1}\right)\right\|_{r}^{o v}\right| \leq K\left|\left\|\chi\left(\tau, x_{2}\right)\right\|_{r}-\left\|\chi\left(\tau, x_{1}\right)\right\|_{r}\right|
$$

since for $|\varrho v|>1$ the power function is locally Lipschitz continuous on $\mathbb{R}^{n}$ with the Lipschitz constant $K$ in $B_{r}\left(\frac{b}{a}\right) \backslash\{0\}$. In addition, there exists some constant $M$ such that

$$
\sup _{t \in\left[0, T_{q}^{\max }(1)\right]}\left\{\frac{\left(\kappa_{2}-\kappa_{1}\right) t^{o}}{\left(\left\|\chi\left(\tau, x_{2}\right)\right\|_{r}^{o v} t^{o}+\kappa_{2}\right)\left(\left\|\chi\left(\tau, x_{1}\right)\right\|_{r}^{o v} t^{o}+\kappa_{2}\right)}\right\} \leq M
$$

hence,

$$
\left|V\left(x_{1}\right)-V\left(x_{2}\right)\right| \leq \sup _{t \in\left[0, T_{q}^{\max }(1)\right]}\left|\left\|\chi\left(t, x_{1}\right)\right\|_{r}-\left\|\chi\left(t, x_{2}\right)\right\|_{r}\right|+\frac{b}{a} M K \sup _{t \in\left[0, T_{q}^{\max }(1)\right]}\left|\left\|\chi\left(t, x_{1}\right)\right\|_{r}-\left\|\chi\left(t, x_{2}\right)\right\|_{r}\right| .
$$


Moreover, under Assumption 1 and following Lemma 1, the trajectories $\chi\left(t, x_{0}\right)$ are locally Lipschitz continuous w.r.t. the initial condition $x_{0}$ and the $r$-homogeneous norm $\|\cdot\|_{r}$ is also locally Lipschitz continuous. Therefore,

$$
\left|V\left(x_{1}\right)-V\left(x_{2}\right)\right| \leq L_{2}\left\|x_{2}-x_{1}\right\|+\frac{b}{a} M K L_{2}\left\|x_{2}-x_{1}\right\|
$$

guaranteeing that the function (20) is locally Lipschitz continuous on $S_{r}(1)$ and by homogeneity it inherits this property for all $\mathbb{R}^{n} \backslash\{0\}$. Using the semi-group property of the state transition map $\chi$ we obtain that $\left\|\chi\left(\tau, \chi\left(t, x_{0}\right)\right)\right\|_{r}=\left\|\chi\left(\tau+t, x_{0}\right)\right\|_{r}$, for all $t, \tau \in \mathbb{R}_{+}$and $x_{0} \in \mathbb{R}^{n}$. In addition,

$$
k\left(\tau, \chi\left(t, x_{0}\right)\right)=\frac{\left\|\chi\left(\tau, \chi\left(t, x_{0}\right)\right)\right\|_{r}^{\rho v} \tau^{\rho}+\kappa_{1}}{\left\|\chi\left(\tau, \chi\left(t, x_{0}\right)\right)\right\|_{r}^{\rho v} \tau^{o}+\kappa_{2}}=\frac{\left.\| \chi\left(\tau+t, x_{0}\right)\right) \|_{r}^{o v} \tau^{o}+\kappa_{1}}{\left.\| \chi\left(\tau+t, x_{0}\right)\right) \|_{r}^{o v} \tau^{o}+\kappa_{2}}
$$

Then, for any $x_{0} \in \mathbb{R}^{n}$, the function (20) satisfies

$$
\begin{aligned}
V\left(\chi\left(t, x_{0}\right)\right) & =\sup _{\tau \geq 0}\left\{\left\|\chi\left(\tau, \chi\left(t, x_{0}\right)\right)\right\|_{r} k\left(\tau, \chi\left(t, x_{0}\right)\right)\right\} \\
& =\sup _{\tau \geq 0}\left\{\left\|\chi\left(\tau+t, x_{0}\right)\right\|_{r} \frac{\left\|\chi\left(\tau+t, x_{0}\right)\right\|_{r}^{\rho v} \tau^{\rho}+\kappa_{1}}{\left\|\chi\left(\tau+t, x_{0}\right)\right\|_{r}^{\rho v} \tau^{\rho}+\kappa_{2}}\right\} \\
& =\sup _{\tau \geq t}\left\{\left\|\chi\left(\tau, x_{0}\right)\right\|_{r} \frac{\left\|\chi\left(\tau, x_{0}\right)\right\|_{r}^{\rho v}(\tau-t)^{\rho}+\kappa_{1}}{\left\|\chi\left(\tau, x_{0}\right)\right\|_{r}^{\rho v}(\tau-t)^{\rho}+\kappa_{2}}\right\} \\
& <\sup _{\tau \geq t}\left\{\left\|\chi\left(\tau, x_{0}\right)\right\|_{r} \frac{\left\|\chi\left(\tau, x_{0}\right)\right\|_{r}^{\rho v} \tau^{\varrho}+\kappa_{1}}{\left\|\chi\left(\tau, x_{0}\right)\right\|_{r}^{\rho v} \tau^{\varrho}+\kappa_{2}}\right\} \\
& <\sup _{\tau \geq 0}\left\{\left\|\chi\left(\tau, x_{0}\right)\right\|_{r} k\left(\tau, x_{0}\right)\right\}=V\left(x_{0}\right),
\end{aligned}
$$

where the strict inequality follows from the strict monotonicity of function $k$, i.e. $k\left(t_{1}, \cdot\right)<k\left(t_{2}, \cdot\right)$ for $t_{1}<t_{2}$. The upper Dini derivative of $V\left(\chi\left(t, x_{0}\right)\right)$ along a trajectory $\chi\left(t, x_{0}\right)$ at the point $x_{0}$ is given by

$$
D^{+} V\left(x_{0}\right) F\left(x_{0}\right)=\limsup _{h \rightarrow 0^{+}} \frac{1}{h}\left[V\left(\chi\left(h, x_{0}\right)\right)-V\left(x_{0}\right)\right],
$$

almost everywhere. Since $V\left(\chi\left(\tau, x_{0}\right)\right)<V\left(x_{0}\right)$ for (small) $\tau>0$ have been proven, then $D^{+} V\left(x_{0}\right) F\left(x_{0}\right)<0$ is deduced for almost all $x_{0} \in \mathbb{R}^{n} \backslash\{0\}$. This concludes the proof of Theorem 3 .

Remark 2. Note that when $q \rightarrow \infty$ the time $T_{q}(x)$ becomes the convergence time, and the Lyapunov function (11) proposed by Theorem 2 corresponds to the case of Massera ${ }^{4}$, where the trajectory is integrated for all the time until it reaches the origin, while the Lyapunov function (19) proposed by Theorem 3 corresponds to the case of Persidskii ${ }^{5}$, where the supremum of the trajectories is looked for all the time until the origin is reached.

Both proposals of computing the Lyapunov functions (11) and (19) up to the times $T_{q}^{\max }\left(\|x\|_{r}\right)$ and $T_{q}(x)$, respectively, can be readily applied for the case of systems with positive homogeneity degree, studied by Efimov et al. ${ }^{11}$ but using $\bar{T}_{q}\left(\|x\|_{r}\right)$. However, the time $T_{q}(x)$ (and $T_{q}^{\max }\left(\|x\|_{r}\right)$ defined as the supremum of $T_{q}(x)$ for all $x$ in the same sphere) greatly simplifies 
the calculations because it is easier to identify when the trajectories $\chi(t, x)$ reach the sphere of radius $\frac{1}{q}\|x\|_{r}$ for the first time, in contrast to the time $\bar{T}_{q}\left(\|x\|_{r}\right)$ from which the trajectories $\chi(t, x)$ never left the ball of radius $\frac{1}{q}\|x\|_{r}$.

\section{5 | NUMERICAL DESIGN}

The Lyapunov functions provided by Theorems 2 and 3 can be numerically realized by following the methodology proposed by Efimov et al. ${ }^{11}$.This procedure is described as follows.

First of all, due to homogeneity properties of the Lyapunov functions proposed in Theorems 2 and 3, for any $x \in \mathbb{R}^{n}$ there is a unique $y \in S_{r}(1)$ such that $x=\Lambda_{r}\left(\|x\|_{r}\right) y$ and $U(x)=\|x\|_{r}^{\rho_{0}} U(y)$, where $\rho_{0}$ is the homogeneity degree of the Lyapunov function. Thus, it is enough to approximate the values of the Lyapunov function on the unit sphere, and later recover its values globally applying the dilation $\Lambda_{r}\left(\|x\|_{r}\right)$.

The methodology of numerical construction of $r$-homogeneous Lyapunov functions presented by Efimov et al. ${ }^{11}$, consists of two steps:

1. Point-wise calculation of some values of the Lyapunov function, uniformly distributed on the unit sphere $S_{r}(1)$, and

2. Interpolation of the Lyapunov function for any $y \in S_{r}(1)$.

This methodology was developed for numerical construction of $r$-homogeneous Lyapunov functions for system with positive homogeneity degree. Nevertheless, an extension to the case of systems with negative homogeneity degree can be easily formulated because the Lyapunov functions provided in the present paper by Theorems 2 and 3 satisfy the properties of homogeneity and Lipschitz continuity required by the proposal of ${ }^{11}$. This extension is developed as follows.

Consider the explicit Euler method ${ }^{30}$. Recall, from Assumptions 1, $\chi\left(t, x_{0}\right)$ is the unique solution of system (1) with initial condition $x_{0}$. So, for a given fixed sampling step $h>0$, the discrete trajectory $\chi_{h}\left(t_{i}, x_{0}\right)$ denotes an approximation at instants $t_{i}=i h, i=1,2,3, \ldots$ of the solution $\chi\left(t, x_{0}\right)$ of $(1)$.

For $N>0$, let $\xi_{j} \in S_{r}(1)$ (with $j=1, \ldots, N$ ) form a uniform grid on the unit sphere. First, considering the Lyapunov function (11) based on the integral of the system's trajectories, define

$$
\begin{gathered}
U_{j}^{h}=h \sum_{i=1}^{J^{\max }}\left\|\chi_{h}\left(t_{i}, \xi_{j}\right)\right\|_{r}^{\mu}, \\
\boldsymbol{J}^{\max }=\arg \inf _{i \geq 0} \sup _{1 \leq j \leq N}\left\|\chi_{h}\left(t_{i}, \xi_{j}\right)\right\|_{r} \leq q^{-1} .
\end{gathered}
$$


Now, considering the Lyapunov function (19) based on the supremum of the system's trajectories:

$$
\begin{gathered}
V_{j}^{h}=\sup _{i \in[0, J]}\left\{\left\|\chi_{h}\left(t_{i}, \xi_{j}\right)\right\|_{r} k\left(t_{i}, \xi_{j}\right)\right\}, \\
J=\arg \inf _{i \geq 0}\left\|\chi_{h}\left(t_{i}, \xi_{j}\right)\right\|_{r} \leq q^{-1},
\end{gathered}
$$

and

$$
k_{j}\left(t_{i}, \xi_{j}\right)=\frac{\left\|\chi_{h}\left(t_{i}, \xi_{j}\right)\right\|_{r}^{\rho v} t_{i}^{\varrho}+\kappa_{1}}{\left\|\chi_{h}\left(t_{i}, \xi_{j}\right)\right\|_{r}^{\rho v} t_{i}^{\varrho}+\kappa_{2}}
$$

where $\varrho>0$ such that $|\varrho v|>1$.

Remark 3. The parameter $J$ in the proposal of Efimov et al. ${ }^{11}$ is taken to warranty that $\left\|\chi_{h}\left(t_{i}, \xi_{j}\right)\right\|_{r} \leq q^{-1}$ holds for all $1 \leq j \leq N$ and all $i \geq J$. However, in that case, it is not clear how to find $J$ at least that the whole trajectory is computed until it converges to the origin. In contrast, our proposal simplifies the calculation of $J$ because it is possible to determine the point when $\left\|\chi_{h}\left(t_{i}, \xi_{j}\right)\right\|_{r} \leq q^{-1}$ holds for the first time and take $J^{\text {max }}$ as the largest $J$ for the initial conditions $\xi_{j} \in S_{r}(1)$ (with $j=1, \ldots, N)$. Moreover, the numerical design of Lyapunov function with the definition of $J$ presented here is more efficient since it reduces the data load. Pleasantly, our proposal can be used for systems with positive homogeneity degree, which were studied by Efimov et al. ${ }^{11}$.

Corollary 2. Let Assumption 1 be satisfied by the system (1). Then there exist $q>1, N>0$ and $h>0$, such that,

$$
U_{j}^{h}=\tilde{U}\left(\xi_{j}\right) \quad \text { and } \quad V_{j}^{h}=\tilde{V}\left(\xi_{j}\right)
$$

for all $j=1, \ldots, N$, where $\tilde{U}: \mathbb{R}^{n} \rightarrow \mathbb{R}_{+}$and $\tilde{V}: \mathbb{R}^{n} \rightarrow \mathbb{R}_{+}$are locally Lipschitz continuous and $r$-homogeneous Lyapunov functions of degree $\mu-v$ and 1 , respectively, for the system (1).

Proof. Since all conditions of Theorems 2 and 3 are satisfied, for $q>1$ sufficiently large a Lyapunov function for the system (1) can be selected in the same form of $U$ given by (11) or $V$ given by (19). By the properties of the (explicit) Euler method ${ }^{30}$, for a sufficiently large value of $N>0$ and a small enough $h>0$ the inequalities

$$
\left|U_{j}^{h}-U\left(\xi_{j}\right)\right|<\epsilon_{1}, \quad \text { and } \quad\left|V_{j}^{h}-V\left(\xi_{j}\right)\right|<\epsilon_{2}
$$

hold for any $\epsilon_{1}, \epsilon_{2}>0$ and all $j=1, \ldots, N$. From Proposition (2), a $r$-homogeneous Lyapunov function possesses certain robustness against small deformations. So, since the values of $\epsilon_{1}, \epsilon_{2}$ can be made arbitrarily small by increasing $N$ and decreasing $h$, if $U$ in (11) and $V$ in (19) are Lyapunov functions for the system (1), then the same is true for a $\tilde{U}$ and $\tilde{V}$ described in Corollary 2 .

Corollary 2 shows that pointwise values of Lyapunov functions $\tilde{U}$ and $\tilde{V}$ can be computed on $S_{r}(1)$ by using the discrete functions (21) and (22), respectively. The next step is to make an interpolation, which is described in ${ }^{11}$ as follows. First, note 
that a homogeneous norm is any positive definite, Lipschitz continuous and homogeneous function of degree 1, such that, $S_{r}(1)=\mathbb{S}^{n}=\left\{x \in \mathbb{R}^{n}:\|x\|=1\right\}$ (for example, an implicit definition of a canonical homogeneous norm from ${ }^{31}$ can be used); then for any $x, y \in \mathbb{S}^{n}$, let $g(x, y)=\arccos \left(x^{\top} y\right)$ denotes the geodesic distance on the unit sphere $\mathbb{S}^{n}$. From the theory of radial basis function interpolation on the sphere ${ }^{32,33}$, a continuous function $p:[0, \pi] \rightarrow \mathbb{R}$ is (zonal) strictly positive definite on $\mathbb{S}^{n}$ if for all distinct points $\xi_{j} \in \mathbb{S}^{n}$ with $j=1, \ldots, N$ and for all $N>0$, the matrix

$$
\Pi_{N}=\left\{p\left(g\left(\xi_{i}, \xi_{j}\right)\right)\right\}_{i, j=1}^{N}
$$

is positive definite. Then selecting a zonal strictly positive definite function $p$ (several examples are provided in, ${ }^{32,33}$ e.g., $p(s)=e^{-a s^{2}}, p(s)=s^{3}$, etc. $)$ :

- There exists always $\theta_{U} \in \mathbb{R}^{N}$ such that $U_{j}^{h}=\tilde{U}\left(\xi_{j}\right)$ for all $j=1, \ldots, N$ where

$$
\tilde{U}(\xi)=\sum_{j=1}^{N} \theta_{U, j} p\left(g\left(\xi, \xi_{j}\right)\right) .
$$

where the vector $\theta_{U}$ is the solution of the equation $\Gamma_{N}^{U}=\Pi_{N} \theta_{U}$, where $\Gamma_{N}^{U}=\left[U_{1}^{h}, \ldots, U_{N}^{h}\right]^{T} \in \mathbb{R}^{N}$.

- There exists always $\theta_{V} \in \mathbb{R}^{N}$ such that $V_{j}^{h}=\tilde{V}\left(\xi_{j}\right)$ for all $j=1, \ldots, N$ where

$$
\tilde{V}(\xi)=\sum_{j=1}^{N} \theta_{V, j} p\left(g\left(\xi, \xi_{j}\right)\right) .
$$

where the vector $\theta_{V}$ is the solution of the equation $\Gamma_{N}^{V}=\Pi_{N} \theta_{V}$, where $\Gamma_{N}^{V}=\left[V_{1}^{h}, \ldots, V_{N}^{h}\right]^{T} \in \mathbb{R}^{N}$.

The matrix $\Pi_{N}$ is non-singular, symmetric and positive definite thanks to the properties of the function $p$. Some assumptions on $p$ and $\Pi_{N}$ are needed:

Assumption 2. The function $p\left(g\left(\xi, \xi_{0}\right)\right)$ is locally Lipschitz continuous with respect to $\xi$ for any $\xi, \xi_{0} \in \mathbb{S}^{n}$.

Assumption 3. There exist $\alpha>0$ and $\rho>0$ such that for any $N>0$

$$
\left\|\Pi_{N}^{-1}\right\|_{2} \leq \frac{\varrho}{N^{1+\alpha}}
$$

where $\|\cdot\|_{2}$ is the induced matrix norm.

In the paper of Efimov et al. ${ }^{11}$, Theorem 3, it has been proven that a locally Lipschitz and $r$-homogeneous Lyapunov function for a $r$-homogeneous system with positive degree is given on the unit sphere $S_{r}(1)$ in the form of (23) or (24). From the results of Corollary 2 and Theorems 2 and 3, the Lyapunov functions (11), (19), (23) and (24) have the properties of boundedness, homogeneity and Lipschitz continuity required by the proof of Theorem $3 \mathrm{in}^{11}$. Therefore, for a large enough $N>0$ (i.e., a sufficiently dense grid on the unit sphere) and a small enough sampling step $h>0,(23)$ appropriately approximates the Lyapunov function (11) on the unit sphere $S_{r}(1)$, whereas (24) appropriately approximates the Lyapunov function (19) on the 
unit sphere $S_{r}(1)$, hence by the robustness of a homogeneous Lyapunov function predicted by Proposition 2, it can be concluded that (23) and (24) are projections on the unit sphere $S_{r}(1)$ of Lyapunov functions for the system (1). Moreover, as it was mentioned before, for any $x \in \mathbb{R}^{n}$ there is a unique $\xi \in S_{r}(1)$ such that $x=\Lambda_{r}\left(\|x\|_{r}\right) \xi$ and $U(x)=\|x\|_{r}^{\rho_{0}} U(\xi)$, where $\rho_{0}$ is the homogeneity degree of the Lyapunov function. Consequently, the following corollary is derived.

Corollary 3. Let Assumptions 1,2 and 3 be satisfied. Then there exist $q>1, N>0$ and $h>0$ such that for any $x \in \mathbb{R}^{n}$ :

- A locally Lipschitz continuous and $r$-homogeneous of degree $\mu-v$ Lyapunov function for the system (1) is given by

$$
\tilde{U}(x)=\|x\|_{r}^{\mu-v} \tilde{U}(\xi)
$$

where $\tilde{U}$ is given by (23).

- A locally Lipschitz continuous and $r$-homogeneous of degree 1 Lyapunov function for the system (1) is given by

$$
\tilde{V}(x)=\|x\|_{r} \tilde{V}(\xi)
$$

where $\tilde{V}$ is given by (24).

Note that by construction, the functions (25) and (26) are positive definite, Lipschitz continuous and $r$-homogeneous of the corresponding degree. To confirm that the methodology was successfully realized, the negative definiteness of the derivative of the Lyapunov function has to be tested. Since the system (1) and the designed Lyaunov functions are $r$-homogeneous of degree $\rho_{0}$ where $\rho_{0}=\mu-v$ or $\rho_{0}=1$, respectively, the verification of the negative definiteness of their derivatives can be performed on the unit sphere $S_{r}(1)$ only, that is, for any $x \in \mathbb{R}^{n}$ there exists an unique $y \in S_{r}(1)$ such that $x=\Lambda_{r}\left(\|x\|_{r}\right) y$, then considering $V$ as a $r$-homogeneous and locally Lipschitz continuous Lyapunov function:

$$
D^{+} V(x) F(x)=\|x\|_{r}^{\rho_{0}} D^{+} V(y) F(y)
$$

The main drawback of the present procedure of numerical design of Lyapunov functions is the selection of the parameters $q>1, N>0$ and $h>0$. There is no way to determine a priori their suitable values yet. Thus, the procedure should be repeated for different values of $q>1, N>0$, and $h>0$ till obtaining a desired Lyapunov function.

\section{6 | EXAMPLES OF NUMERICAL DESIGN OF LYAPUNOV FUNCTIONS}

As it has been mentioned before, many HOSM algorithms satisfy Assumption 1, hence a Lyapunov function like (25) or (26) can be computed for them by following the methodology described in Section 5. To illustrate the methodology developed along with the paper two examples are provided: the Twisting Algorithm for the design based on the integral of the trajectories and the 


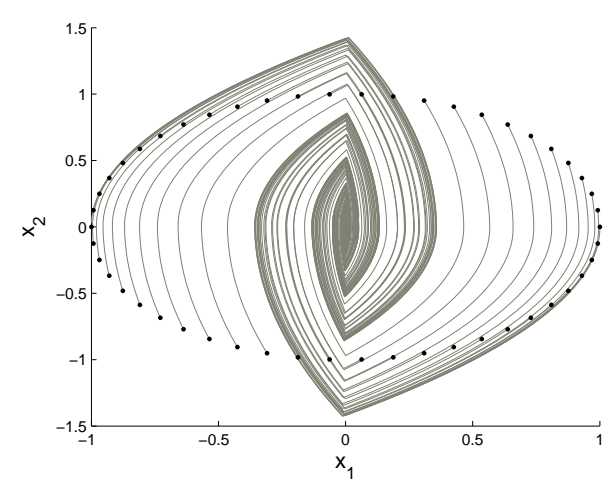

(a) Vector field.

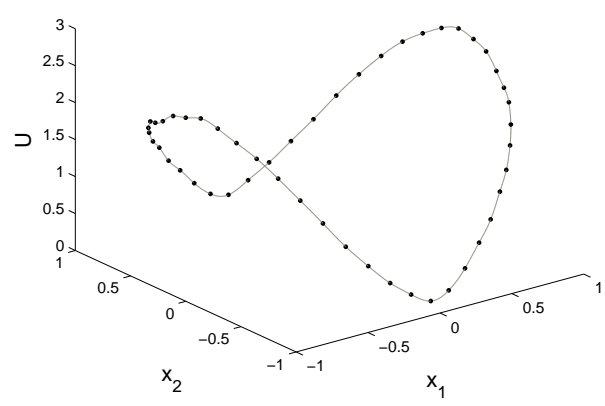

(b) Lyapunov function on the unit sphere.

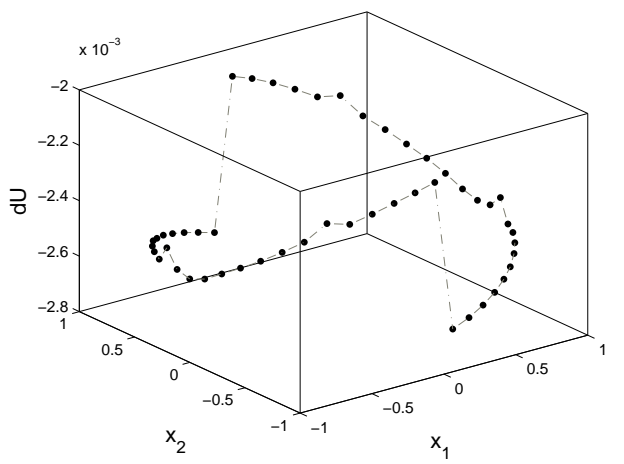

(c) Derivative of Lyapunov function on the unit sphere.

FIGURE 1 : Results of numerical design of a Lyapunov function for the system (27).

(Nonsingular) Quasi-continuous Control Algorithm for the design based on the supremum of the trajectories. Both algorithms are described in detail by the book of Shtessel et al. ${ }^{16}$

\section{1 | Design based on the integral of the trajectories}

Consider the Twisting Algorithm given by

$$
\dot{x}_{1}=x_{2}, \quad \dot{x}_{2}=-c_{1}\left\lceil x_{1}\right\rfloor^{0}-c_{2}\left\lceil x_{2}\right\rfloor^{0},
$$


where $x \in \mathbb{R}^{2}$ is the state and $c_{1}, c_{2} \in \mathbb{R}_{+}$have to satisfy $c_{1}>c_{2}$ to warranty stability at the origin. The system (27) is $r$-homogeneous of degree $v=-1$ and weights $r=[2,1]$. From Corollary 3 and considering the parameters and expressions:

$$
\begin{gathered}
q=10, \quad h=0.01, \quad a=50, \quad \mu=3, \quad N=50, \quad c_{1}=2, \quad c_{2}=1, \\
p(s)=e^{-a s^{2}}, \quad g(x, y)=\arccos \left(x^{\top} y\right),
\end{gathered}
$$

a Lyapunov function for the system (27) is given by

$$
\tilde{U}(x)=\|x\|_{r}^{\mu-\nu} \sum_{j=1}^{N} \theta_{j} e^{-a \arccos ^{2}\left(\xi^{\top} \xi_{j}\right)}
$$

where $\xi \in S_{r}(1)$ such that $x=\Lambda_{r}\left(\|x\|_{r}\right) \xi$ and the points $\xi_{j} \in S_{r}(1)$, with $j=1, \ldots, N$, form an uniform grid on the unique sphere. Moreover, the vector $\theta$ is the solution of the equation $\Gamma_{N}=\Pi_{N} \theta$, where $\Pi_{N}=\left\{e^{-a \arccos { }^{2}\left(\xi_{i}^{\top} \xi_{j}\right)}\right\}_{i, j=1}^{N}$ and $\Gamma_{N}=$ $\left[U_{1}^{h}, \ldots, U_{N}^{h}\right]^{T}$ where $U_{j}^{h}$ are given by (21) with $\mu=3$. Furthermore, the system's trajectories $\chi_{h}\left(t_{i}, \xi_{j}\right)$, with $j=1, \ldots, N$, are computed by using the Euler method ${ }^{30}$ with $h=0.01$.

Figure 1 presents the results of the numerical design of a Lyapunov function for the system (27), Fig. 1a shows system's trajectories where the black points are the initial data. Note that there is not sliding motion on the interval of time where the trajectories were computed. Furthermore, Fig. 1b provides the values of the designed Lyapunov function (28) on the unit sphere. The black points represents the components of the vector $\Gamma_{N}=\left[U_{1}^{h}, \ldots, U_{N}^{h}\right]^{T}$ and the grey line represents the interpolation. Finally, a point-wise calculation of the derivative of the Lyapunov function is provided in Fig. (1c) depicted by the black marks and the dash-dot grey line is just a reference to joint the points and highlight the plot.

\section{2 | Design based on the supremum of the trajectories}

Consider the (nonsingular) Quasi-continuous Control Algorithm given by

$$
\dot{x}_{1}=x_{2}, \quad \dot{x}_{2}=-\alpha \frac{\beta x_{1}+\left\lceil x_{2}\right\rfloor^{2}}{\beta\left|x_{1}\right|+\left|x_{2}\right|^{2}}
$$

where $x \in \mathbb{R}^{2}$ is the state and $\alpha, \beta>0$ are tunning parameters. The system (29) is $r$-homogeneous of degree $v=-1$ and weights $r=[2,1]$. From Corollary 3 and considering the parameters and expressions:

$$
\begin{gathered}
q=3, \quad h=0.01, \quad a=50, \\
\rho=2, \quad \kappa_{1}=1, \quad \kappa_{2}=2, \\
N=50, \quad \alpha=3, \quad \beta=5, \\
p(s)=e^{-a s^{2}}, \quad g(x, y)=\arccos \left(x^{\top} y\right),
\end{gathered}
$$




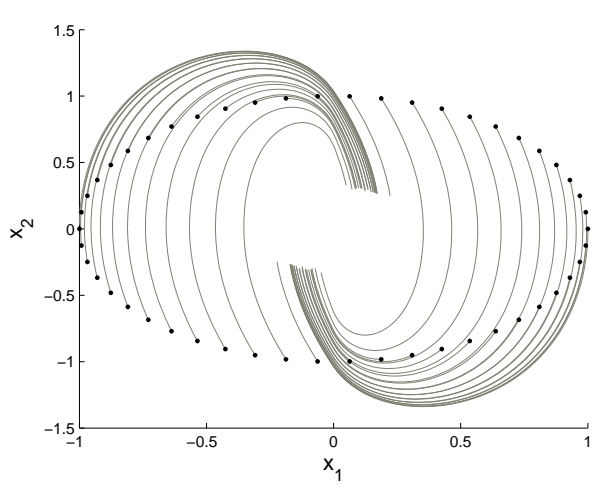

(a) Vector field.

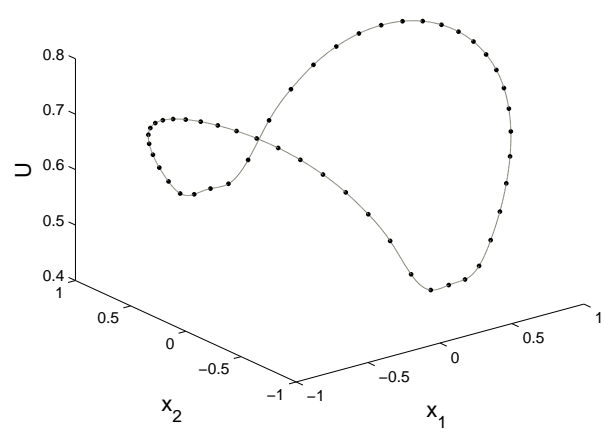

(b) Lyapunov function on the unit sphere.

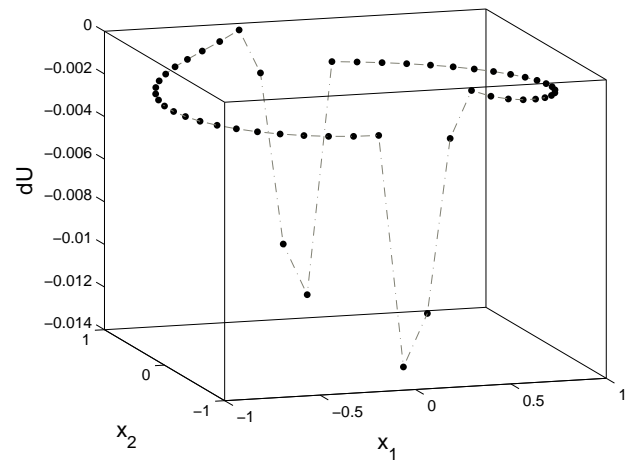

(c) Derivative of Lyapunov function on the unit sphere.

FIGURE 2 : Results of numerical design of a Lyapunov function for the system (29).

a Lyapunov function for the system (29) is given by

$$
\tilde{V}(x)=\|x\|_{r} \sum_{j=1}^{N} \theta_{j} e^{-a \arccos ^{2}\left(\xi^{\top} \xi_{j}\right)}
$$

where $\xi \in S_{r}(1)$ such that $x=\Lambda_{r}\left(\|x\|_{r}\right) \xi$ and the points $\xi_{j} \in S_{r}(1)$, with $j=1, \ldots, N$, form an uniform grid on the unique sphere. Moreover, the vector $\theta$ is the solution of the equation $\Gamma_{N}=\Pi_{N} \theta$, where $\Pi_{N}=\left\{e^{-a \arccos ^{2}\left(\xi_{i}^{\top} \xi_{j}\right)}\right\}_{i, j=1}^{N}$ and $\Gamma_{N}=\left[V_{1}^{h}, \ldots, V_{N}^{h}\right]^{T}$ where $V_{j}^{h}$ are given by (22) with $\varrho=2, \kappa_{1}=1$ and $\kappa_{2}=2$. Furthermore, the system trajectories $\chi_{h}\left(t_{i}, \xi_{j}\right)$, with $j=1, \ldots, N$, are computed by using the Euler method ${ }^{30}$ with $h=0.01$. The numerical design of a Lyapunov function 
for the system (29) is shown by Fig. 2. The system's solutions are presented in Fig. 2a where the black points are the initial conditions, note that they do not exhibit sliding motion. Moreover, the values of the designed Lyapunov function (30) on the unit sphere are depicted by Fig. 2b, where the black points represents the components of the vector $\Gamma_{N}=\left[U_{1}^{h}, \ldots, U_{N}^{h}\right]^{T}$ and the grey line represents the interpolation. Finally, a point-wise calculation of the derivative of the Lyapunov function is provided in Fig. (2c) highlighted by the black marks and the dash-dot grey line is just a reference to joint the points.

\section{7 | CONCLUSIONS}

This paper was devoted to the construction of homogeneous and Lipschitz continuous Lyapunov functions for a class of piecewise continuous and homogeneous systems of negative degree. The presented converse Lyapunov theorems extend classical results about Lyapunov function design (such as Kurzweil, Massera, Persidskii, and Yoshizawa) to the considered class of systems, including some well-known HOSM algorithms. Moreover, explicit expressions of the Lyapunov function were provided, the first one based on the integral of the system's trajectories and the second one on their supremum. It was shown that such expressions of Lyapunov functions could be numerically realized under some technical assumptions. Finally, such a methodology of a numerical Lyapunov function design was illustrated by considering two HOSM algorithms.

\section{References}

1. Bacciotti A, Rosier L. Lyapunov functions and stability in control theory. Volume 267 of Lecture Notes in Control and Information Sciences. Berlin, Germany: Springer-Verlag. 2nd ed. 2005.

2. Zubov VI. Methods of A.M. Lyapunov and their application. Groningen, Netherlands: Popko Noordhoff . 1964.

3. Kurzweil J. On the inversion of Lyapunov's second theorem on stability of motion. AMS Translations Series 2 1963; 24 : 19-77.

4. Massera JL. On Lyapunov's conditions of stability. Annals of Mathematics 1949; 50: 705-721.

5. Persidskii KP. On a theorem of Lyapunov. C. R. Dokl. Acad. Sci. URSS 1937: 541-544.

6. Yoshizawa T, others. On the stability of solutions of a system of differential equations. Memoirs of the College of Science, University of Kyoto. Series A: Mathematics 1955; 29(1): 27-33.

7. Clarke FH, Ledyaev YS, Stern RJ. Asymptotic stability and smooth Lyapunov functions. Journal of differential Equations 1998; 149(1): 69-114. 
8. Rosier L. Smooth Lyapunov functions for discontinuous stable systems. Set-Valued Analysis 1999; 7(4): 375-405.

9. Kellett CM, Teel AR. Smooth Lyapunov functions and robustness of stability for difference inclusions. Systems \& Control Letters 2004; 52(5): 395-405.

10. Rosier L. Homogeneous Lyapunov function for homogeneous continuous vector field. Systems \& Control Letters 1992; 19(6): 467-473.

11. Efimov D, Ushirobira R, Moreno JA, Perruquetti W. Homogeneous Lyapunov Functions: From Converse Design to Numerical Implementation. SIAM Journal on Control and Optimization 2018; 56(5): 3454-3477.

12. Sanchez T, Moreno JA. Design of Lyapunov functions for a class of homogeneous systems: Generalized forms approach. International Journal of Robust and Nonlinear Control 2019; 29(3): 661-681.

13. Sánchez T, Moreno JA. Construction of Lyapunov Functions for High Order Sliding Modes. In: Fridman L, Barbot JP, Plestan F., eds. Recent Trends in Sliding Mode ControlLondon, UK: The Institution of Engineering and Technology (IET). 2016 (pp. 77-99).

14. Nakamura H, Yamashita Y, Nishitani H. Smooth Lyapunov functions for homogeneous differential inclusions. In: Proceedings of the 41st SICE Annual Conference. IEEE. ; 2002; Osaka, Japan: 1974-1979.

15. Tuna SE, Teel AR. Homogeneous hybrid systems and a converse Lyapunov theorem. In: Proceedings of the 45th IEEE Conference on Decision and Control (CDC). IEEE. ; 2006; San Diego, CA, USA: 6235-6240.

16. Shtessel Y, Edwards C, Fridman L, Levant. A. Sliding Mode Control and Observation. New York, NY, USA: Brikhauser: Basel . 2014.

17. Moreno JA, Osorio M. Strict Lyapunov functions for the super-twisting algorithm. IEEE Transactions on Automatic Control 2012; 57(4): 1035-1040.

18. Cruz-Zavala E, Moreno JA. Homogeneous high order sliding mode design: a Lyapunov approach. Automatica 2017; 80: 232-238.

19. Polyakov A, Poznyak A. Lyapunov function design for finite-time convergence analysis:“Twisting” controller for secondorder sliding mode realization. Automatica 2009; 45(2): 444-448.

20. Polyakov A, Poznyak A. Unified Lyapunov function for a finite-time stability analysis of relay second-order sliding mode control systems. IMA Journal of Mathematical Control and Information 2012; 29(4): 529-550. 
21. Polyakov A, Efimov D, Perruquetti W. Finite-time and fixed-time stabilization: Implicit Lyapunov function approach. Automatica 2015; 51: 332-340.

22. Sánchez T, Moreno JA. Construction of lyapunov functions for a class of higher order sliding modes algorithms. In: Proceedings of the 51st IEEE Conference on Decision and Control (CDC). IEEE. ; 2012; Maui, HI, USA: $6454-6459$.

23. Sánchez T, Moreno JA. Lyapunov functions for Twisting and Terminal controllers. In: Proceedings of the 13th International Workshop on Variable Structure Systems (VSS). IEEE. ; 2014; Nantes, France: 1-6.

24. Filippov AF. Differential equations with discontinuous right-hand side. Dordrecht, Netherlands: Kluwer Academic Publishers . 1988.

25. Cortes J. Discontinuous dynamical systems. IEEE Control Systems Magazine 2008; 28(3): 36-73.

26. Bernuau E, Efimov D, Perruquetti W, Polyakov A. On an extension of homogeneity notion for differential inclusions. In: Proceedings of the 2013 European Control Conference (ECC). IEEE. ; 2013; Zürich, Switzerland: 2204-2209

27. Levant A, Efimov D, Polyakov A, Perruquetti W. Stability and robustness of homogeneous differential inclusions. In: Procedings of the 55th IEEE Conference on Decision and Control (CDC). IEEE. ; 2016; Las Vegas, NV, USA: 7288-7293.

28. Polyakov A. Nonlinear Feedback Design for Fixed-Time Stabilization of Linear Control Systems. IEEE Transactions on Automatic Control 2012; 57(8): 2106-2110.

29. Bhat SP, Bernstein DS. Geometric homogeneity with applications to finite-time stability. Mathematics of Control, Signals and Systems 2005; 17(2): 101-127.

30. Butcher JC. Numerical methods for ordinary differential equations. New York, NY, USA: John Wiley \& Sons . 2003.

31. Polyakov A, Coron J, Rosier L. On finite-time stabilization of evolution equations: A homogeneous approach. In: Procedings of the 55th IEEE Conference on Decision and Control (CDC). IEEE. ; 2016; Las Vegas, NV, USA: $3143-3148$.

32. Cheney EW. Approximation and interpolation on spheres. In: Singh S., ed. Approximation Theory, Wavelets and ApplicationsBerlin, Germany: Springer. 1995 (pp. 47-53).

33. Hubbert S. Radial basis function interpolation on the sphere. $\mathrm{PhD}$ thesis. Imperial College London, London, UK; 2002. 